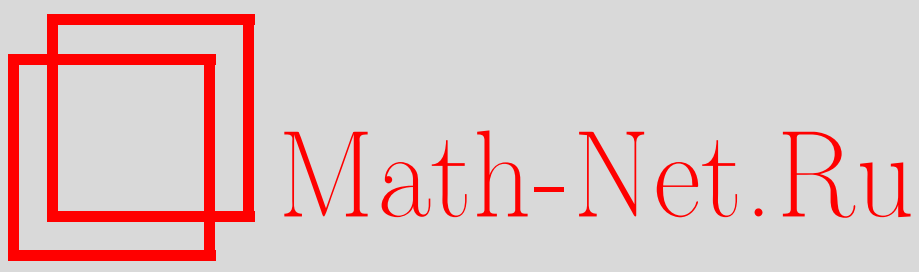

М. И. Кузнецов, Н. Г. Чебочко, Деформации классических алгебр Ли, Матем. сб., 2000, том 191, номер 8, 69-88

DOI: https://doi.org/10.4213/sm499

Использование Общероссийского математического портала Math-Net.Ru подразумевает, что вы прочитали и согласны с пользовательским соглашением

http://www . mathnet.ru/rus/agreement

Параметры загрузки:

IP: 18.234 .156 .22

26 апреля 2023 г., $14: 52: 56$ 


\author{
М.И. Кузнецов, Н.Г. Чебочко
}

\title{
Деформации классических алгебр Ли
}

\begin{abstract}
Для классической алгебры Ли $L$ характеристики $p>2$, отличной от $C_{2}$ при $p=3$, доказывается, что $H^{2}(L, L)=0$. Под классической алгеброй Ли понимается алгебра Ли простой алгебраической группы, либо ее факторалгебра по центру, либо алгебра Ли $A_{l}^{z}, l+1 \equiv 0(p)$, или $E_{6}^{z}$ при $p=3$.

Библиография: 14 названий.
\end{abstract}

\section{§1. Введение}

В настоящей работе изучаются деформации классических алгебр Ли над полем $K$ характеристики $p>2$. Под классической алгеброй Ли над $K$ мы понимаем алгебру Ли простой алгебраической группы либо ее факторалгебру по центру. Кроме того, к классическим алгебрам мы относим алгебры Ли $A_{l}^{z}$, когда $l+1 \equiv 0(p)$, и $E_{6}^{z}$ при $p=3$, которые будут описаны ниже (см. $\left.\S 2\right)$.

Известно [1], что над полем характеристики $p>3$ все классические алгебры Ли являются жесткими. Над полем характеристики 3 корневую систему типа $C_{2}$ могут иметь неизоморфные алгебры Ли (см. [2]). Глобальные деформации алгебры Ли $C_{2}$ построены в [3]. В [4] анонсирован результат, согласно которому алгебра Ли $C_{2}$ единственная среди алгебр Ли серий $A_{n}, B_{n}, C_{n}, D_{n}$, допускаюшая нетривиальные деформации при $p=3$. Полное описание глобальных деформаций алгебры Ли $C_{2}$ получено в [5].

В данной работе доказывается

Теорема. Все классические алгебры Ли над полем $K$ характеристики $p>2$ являются жесткими, кроме алгебры Ли $C_{2}$ при $p=3$.

Жесткость алгебры Ли типа $G_{2}$ при $p=3$ была доказана в работе [6], и в настоящей работе алгебра этого типа исключается из рассмотрения.

Условие $H^{2}(L, L)=0$ является достаточным условием жесткости алгебры Ли $L$ (см. [5], [7]). В работе предлагается следуюшая схема вычисления $H^{2}(L, L)$, пригодная для полей характеристики $p>2$. Группа Aut $L$ содержит присоединенную группу Шевалле $G(L)$ (см. [8]). Мы раскладываем основной комплекс в прямую сумму весовых подкомплексов, используя естественное действие максимального тора группы $G(L)$. Соответствуюшие группы когомологий являются весовыми подпространствами в группе когомологий основного комплекса. В $\S 3$ мы показываем, что $H^{2}(L, L)=H_{0}^{2}(L, L)$.

Работа вьполнена при финансовой поддержке Российского фонда фундаментальных исследований (гранты № № 96-01-01756, 99-01-00398).

(C) М. И. КУЗнецОв, Н. Г. ЧевОчко 2000 
В $\S 4$ доказывается тривиальность $H_{0}^{2}(L, L)$. Для коцикла $\psi$ нулевого веса строится алгебра Ли $L(\varepsilon)$ над алгеброй двойных чисел $K[\varepsilon]$. Алгебра $L(\varepsilon)$ имеет градуировку с коэффициентами в группе $Q(R)$, где $Q(R)$ - подгруппа в евклидовом пространстве, порожденная корнями. Выбирая одну из стандартных градуировок, мы получим градуировку в алгебре $L(\varepsilon)$ глубины 1 или 2 .

Теперь можно применить индукцию по рангу алгебры и считать, что коцикл $\psi$ тривиален на компоненте $L_{0}$ градуировки. Несложные рассуждения показьвают, что исходньй коцикл когомологичен коциклу, для которого $\psi\left(L_{-1}, L_{0}\right)=$ $\psi\left(L_{-1}, L_{-1}\right)=0$. После этого можно применить теорию продолжений Картана. Дело в том, что для каждого элемента $l$ из $L_{1} \psi(l,-)$ принадлежит продолжению Картана $L^{(1)}$ пары $\left(L_{-1}, L_{0}\right)$ и нетривиальность $\psi$ означает наличие еще одного $L_{0}$-подмодуля в $L^{(1)}$, изоморфного $L_{1}$, но отличного от $L_{1}$. Отметим, что именно так возникают деформации $L(\varepsilon)$ в работе [3]. Исследование картановского продолжения показывает, что таких подмодулей не сушествует. Поэтому, заменяя $\psi$ на когомологичный коцикл, можно считать, что $\psi\left(L_{1}, L_{-1}\right)=0$. Из полученных условий следует, что $\psi$ - тривиальный коцикл. Таким образом, $H_{0}^{2}(L, L)=0$.

\section{§2. Классические алгебры Ли}

Пусть $L_{\mathbb{C}}$ - комплексная классическая алгебра Ли, $R$ - ее система корней, $Q(R)$ - подгруппа, порожденная корнями, $W(R)$ - группа Вейля. Через $e_{\alpha}, \alpha \in R$, обозначаются корневые векторы из базиса Шевалле, $h_{\alpha}=\left[e_{\alpha}, e_{-\alpha}\right], L_{\mathbb{Z}}-$ соответствуюшая форма Шевалле. Классическая алгебра Ли $L$ над полем $K$ характеристики $p>0$ определяется как $L_{\mathbb{Z}} \otimes_{\mathbb{Z}} K$. Отметим, что равенство $\left[e_{\alpha}, e_{\beta}\right]=0$ при $\alpha+\beta \in R$ возможно только в алгебре Ли типа $G_{2}$ при $p=3$. Если $L_{\mathbb{C}}$ имеет тип $A_{l}$, $l+1 \equiv 0(p)$, или $E_{6}, p=3$, то алгебра Ли $L$ имеет одномерный центр. Через $\bar{A}_{l}, \bar{E}_{6}$ будем обозначать соответствуюшую факторалгебру по центру. Эти алгебры Ли мы также будем относить к классическим алгебрам Ли. Система корней $R$, группы $W(R), Q(R)$ будут в равной степени относится как к алгебрам Ли $L$, так и к их факторалгебрам по центру. Ранг системы $R$ будет называться рангом алгебры Ли $L$. Отметим, что $R$ является системой векторов в вещественном евклидовом пространстве $V_{\mathbb{R}}$. Элементы из $R$ можно рассматривать как линейные функции на картановской подалгебре $\mathscr{H}$ в $L$ (со значениями в $K)$. Чтобы избежать двусмысленности, мы будем иногда обозначать функцию на $\mathscr{H}$, соответствующую $\alpha \in R$, через $\left.\alpha\right|_{\mathscr{H}}$.

Глобальной деформаиией алгебры Ли $L$ называется семейство алгебр Ли, параметризованных точками связного гладкого многообразия, одной из точек которого соответствует алгебра Ли $L$. Пусть $\mathscr{L}$ - многообразие структур алгебр Ли на векторном пространстве $V$. Алгебра Ли $L \in \mathscr{L}$ называется жесткой, если существует окрестность $L$ (в топологии Зарисского на $\mathscr{L}$ ), все точки которой являются алгебрами Ли, изоморфными $L$.

Орбиты естественного действия группы $G=G L(V)$ на $\mathscr{L}$ соответствуют классам изоморфизма алгебр Ли. Пусть $T_{L}(\mathscr{L})$ - касательное пространство к многообразию $\mathscr{L}$ в точке $L \in \mathscr{L}, T_{L}\left(G_{L}\right)$ - касательное пространство к $G$-орбите точки $L$. Согласно [5] пространство локальных деформаций $H_{\mathrm{loc}}(L)=T_{L}(\mathscr{L}) / T_{L}\left(G_{L}\right)$ является фактором группы когомологий $H^{2}(L, L)$. 
Таким образом, условие $H^{2}(L, L)=0$ является достаточным условием жесткости алгебры Ли $L$.

Обозначим через $Q_{*}(R)$ множество всех $\mu \in Q(R)$ таких, что $\mu(\mathscr{H})=0$. Очевидно, если матрища Картана алгебры $L$ невырождена, то $Q_{*}(R)=p Q(R)$.

Пусть $C^{\bullet}(L, L)$ - стандартный комплекс алгебры Ли $L$. Группа $\operatorname{Aut}(L)$ действует естественным образом на $C^{\bullet}(L, L)$. Разложим $C^{\bullet}(L, L)$ в прямую сумму весовых подкомплексов относительно максимального тора $T$ группы $G(L)$ :

$$
C^{\bullet}(L, L)=\bigoplus_{\mu \in Q(R)} C_{\mu}^{\bullet}(L, L)
$$

Пусть $\Lambda_{i}(R) \subset Q(R)$ - подмножество весов $H^{i}(L, L)$ относительно $T$,

$$
H^{i}(L, L)=\bigoplus_{\mu \in \Lambda_{i}(R)} H_{\mu}^{i}(L, L)
$$

Выбирая базис из корневых векторов в $L$, мы естественным образом получаем базис в $C^{i}(L, L)$. Если $e_{\alpha_{1}}^{*} \wedge \cdots \wedge e_{\alpha_{i}}^{*} \otimes e_{\alpha_{i+1}} \in C_{\mu}^{i}(L, L)$, то $\mu(H)=\left(-\alpha_{1}-\cdots-\alpha_{i}+\right.$ $\left.\alpha_{i+1}\right)(H), H \in \mathscr{H}$. Используя тривиальность действия $L$ на $H^{\bullet}(L, L)$, получаем, что $\Lambda_{i}(R) \subset Q_{*}(R)$.

В дальнейшем нам понадобятся сведения о дискриминанте формы Киллинга в базисе Шевалле. Согласно [9; гл. I, $\S 4$, утверждение 4.8.] дискриминант $\Delta$ формы Киллинга в базисе Шевалле можно вычислить по формуле

$$
\Delta=(-1)^{n}\left(2 m^{*}\right)^{l} c^{n_{1}+n_{2}} f
$$

где $n$ - число положительных корней системы $R, n_{1}$ (соответственно $\left.n_{2}\right)$ - число коротких (соответственно коротких простых) корней, $l$ - размерность алгебры $L$, $c$ - отношение квадрата длины длинного корня к квадрату длины короткого корня, $m^{*}=\sum m_{i}^{*}+1, m_{i}^{*}=m_{i}$, если $\alpha_{i}$ - длинньй корень, и $m_{i}^{*}=m_{i} / c$, если $\alpha_{i}-$ короткий корень, $\sum m_{i} \alpha_{i}-$ разложение максимального корня по простым корням, $f$ - определитель матрицы Картана.

Следующее утверждение хорошо известно в случае нулевой характеристики, но, по-видимому, требует некоторых комментариев в случае характеристики $p>0$.

ПРЕДЛОЖЕНИЕ 1. Если L - алгебра Ли с невырожденной формой Киллингa, mo $H^{2}(L, L)=0$.

ДоКАЗАТЕЛЬСТво основано на применении элемента Казимира $k=\sum_{i=1}^{n} e_{i} e^{i}$, $k \in U(L)$, где $\left\{e_{i}\right\}$ - базис Шевалле в $L,\left\{e^{i}\right\}$ - базис, двойственный к $\left\{e_{i}\right\}$ относительно формы Киллинга. Надо показать, что $k$ действует ненулевым образом на $L$. Для этого рассмотрим алгебру Ли $L_{\mathbb{Q}}$ над полем рациональных чисел с базисом Шевалле $\left\{e_{i}\right\}$ и алгебру $L_{\mathbb{Z}_{(p)}}$ с тем же базисом над локализацией $\mathbb{Z}$ по простому идеалу $(p), L_{\mathbb{Z}_{(p)}} \subset L_{\mathbb{Q}}$.

Так как дискриминант формы Киллинга в базисе Шевалле $\left\{e_{i}\right\}$ является целым числом, взаимно простым с $p$, то элементы двойственного базиса $e^{i}$ лежат в $L_{\mathbb{Z}_{(p)}}$. Поэтому элемент Казимира $k=\sum_{i=1}^{n} e_{i} e^{i} \in U\left(L_{\mathbb{Z}_{(p)}}\right)$. 
Очевидно, классическая алгебра Ли $L$ над полем $K$ характеристики $p$ получается из $L_{\mathbb{Z}_{(p)}}$ расширением скаляров $L=L_{\mathbb{Z}_{(p)}} \otimes_{\mathbb{Z}_{(p)}} K$. Здесь $K$ рассматривается как алгебра над $\mathbb{Z}_{(p)}$ относительно естественного гомоморфизма $\mathbb{Z}_{(p)} \rightarrow \mathbb{F}_{p} \subset K$. Для всякого линейного над $\mathbb{Z}_{(p)}$ оператора $\varphi: L_{\mathbb{Z}_{(p)}} \rightarrow L_{\mathbb{Z}_{(p)}}$ имеем линейный над $K$ оператор $\varphi \otimes 1: L \rightarrow L$.

Как известно, оператор Казимира $k$ действует тождественно на $L_{\mathbb{Q}}$ и, в частности, на $L_{\mathbb{Z}_{(p)}}$. Следовательно, $k \otimes 1$ действует тождественно на $L$. Элементы $e_{i} \otimes 1 \in L=L_{\mathbb{Z}_{(p)}} \otimes_{\mathbb{Z}_{(p)}} K$ образуют базис Шевалле в $L, e^{i} \otimes 1-$ двойственный к нему базис относительно формы Киллинга. Поэтому $k \otimes 1=\left(\sum_{i=1}^{n} e_{i} e^{i}\right) \otimes 1=$ $\sum_{i=1}^{n}\left(e_{i} \otimes 1\right)\left(e^{i} \otimes 1\right)$ - оператор Казимира в $L$. Дальнейшее доказательство предложения проходит так же, как в случае нулевой характеристики.

В дальнейшем мы можем исключить из рассмотрения алгебры с невырожденной формой Киллинга.

Пусть $L$ - алгебра ранга $l, l \geqslant 2, L$ отлична от $G_{2}, B=\left\{\alpha_{1}, \ldots, \alpha_{l}\right\}$ - базис $R$, $L=\mathscr{H} \oplus \bigoplus_{\gamma \in R} L_{\gamma}-$ корневое разложение. В $L$ можно получить градуировку вида

$$
L=L_{-2}+L_{-1}+L_{0}+L_{1}+L_{2}
$$

где $L_{0}=M_{0}+I, M_{0}$ - алгебра классического типа ранга $l-1, \operatorname{dim} I \leqslant 1$. Действительно, пусть $\alpha \in B$ и $\alpha$ связан в схеме Дынкина только с одним из корней в $B$. Положим $L_{0}=\mathscr{H} \oplus \bigoplus_{\gamma \in R_{0}} L_{\gamma}, L_{i}=\bigoplus_{\gamma \in R_{i}} L_{\gamma}, i \in\{-2,-1,1,2\}, R_{i}-$ подмножество корней из $R$, в разложение которых по базису корень $\alpha$ входит с коэффициентом $i$. Корень $\alpha$ можно выбрать таким образом, что $\operatorname{dim} L_{ \pm 2} \leqslant 1$. Если $L$ - алгебра типа $\bar{A}_{l}$ или $\bar{E}_{6}$, то $L_{0}=M_{0}$. В остальных случаях $L_{0}=M_{0}+\left\langle h_{0}\right\rangle$, $h_{0} \in \mathscr{H}$.

Таблица 1 показывает, как это реализуется в конкретных алгебрах. В таблице также приведены данные о форме Киллинга алгебры $L$. Из формулы $(1)$ следует, что при $p>2$ форма Киллинга вырождена на $L$, только если $m^{*} \equiv 0(p)$ или $f \equiv 0(p)$.

ТАБЛИЦА 1

\begin{tabular}{|c|c|c|c|c|}
\hline Тип $R$ & Тип $R_{0}$ & $\begin{array}{c}\text { Глубина } \\
\text { градуировки }\end{array}$ & $m^{*}$ & $f$ \\
\hline$A_{l}$ & $A_{l-1}$ & 1 & $l+1$ & $l+1$ \\
$B_{l}$ & $B_{l-1}$ & 1 & $2 l-1$ & 2 \\
$C_{l}$ & $A_{l-1}$ & 1 & $l+1$ & 2 \\
$D_{l}$ & $D_{l-1}$ & 1 & $2(l-1)$ & 4 \\
$E_{6}$ & $D_{5}$ & 1 & 12 & 3 \\
$E_{7}$ & $D_{6}$ & 2 & 18 & 2 \\
$E_{8}$ & $E_{7}$ & 2 & 30 & 1 \\
$F_{4}$ & $C_{3}$ & 1 & 12 & 1 \\
\hline
\end{tabular}

Отметим, что матрица Картана для $M_{0}$ вырождена только для алгебр $L$ типа $A_{l}, C_{l}, l \equiv 0(p)$. На таких алгебрах форма Киллинга невырождена, так как $l+1 \not \equiv 0(p)$ и, значит, $H^{2}(L, L)=0$. В остальных случаях центр в $L_{0}$ выделяется 
прямым слагаемьм, $L_{0}=M_{0} \oplus\left\langle h_{0}\right\rangle, M_{0}$ - классическая алгебра Ли с невырожденной матрицей Картана. Легко проверяется, что при $p>2 L_{ \pm 1}$ - неприводимые $L_{0}$-модули.

Определим алгебры Ли $A_{l}^{z}, l+1 \equiv 0(p)$, и $E_{6}^{z}, p=3$. Пусть $L$ - одна из алгебр $\bar{A}_{l}, \bar{E}_{6}, L=L_{-1} \oplus L_{0} \oplus L_{1}-$ градуировка в алгебре Ли $L$, описанная выше. Добавляя к $L_{0}$ элемент $z$ такой, что ad $\left.z\right|_{L_{s}}=s \mathrm{id}, s=0, \pm 1$, мы получаем алгебры Ли типов $A_{l}^{z}$ и $E_{6}^{z}$. Эти алгебры Ли мы также относим к классическим. Отметим, что алгебра Ли $L$ типа $A_{l}^{z}$ или $E_{6}^{z}$ имеет градуировку $L=L_{-1}+L_{0}+L_{1}$ и $\langle z\rangle-$ центр $L_{0}$. Иногда мы будем обозначать $z$ через $h_{0}$.

Из формулы (1) следует, что форма Киллинга алгебры Ли типа $G_{2}$ вырождена только при $p=2,3$. Поэтому жесткость алгебры Ли типа $G_{2}$ при $p>3$ следует из предложения 1.

Следующая лемма носит вспомогательный характер и будет использоваться в $§ 4$.

ЛЕмма 1. Пусть $L_{0}$ - нулевая компонента в градуировке алгебры Ли L, $L_{0}=M_{0} \oplus\left\langle h_{0}\right\rangle$. Eсли $H_{0}^{2}\left(M_{0}, M_{0}\right)=0$, mo $H_{0}^{2}\left(L_{0}, L_{0}\right)=0$.

ДокАЗАтЕльство. Для доказательства тривиальности $H_{0}^{2}\left(L_{0}, L_{0}\right)$ применим спектральную последовательность Серра-Хохшильда $\left\{E_{r}^{p, q}\right\}$ для алгебры $L_{0}$, идеала $I=\left\langle h_{0}\right\rangle$ и присоединенного $L_{0}$-модуля. Очевидно, все члены спектральной последовательности инвариантны относительно максимального тора $T$ и, следовательно, раскладьваются на весовые подпространства $E_{r, \mu}^{p, q} \cdot\left\{E_{r, \mu}^{p, q}\right\}$ является спектральной последовательностью Серра-Хохшильда для подкомплекса $C_{\mu}^{\bullet}(L, L)$, сходящейся к $H_{\mu}^{\bullet}(L, L)$. В частности, для $H_{0}^{2}(L, L)$ получаем

$$
\begin{aligned}
& E_{2,0}^{2,0}=H_{0}^{2}\left(L_{0} / I, H^{0}\left(I, L_{0}\right)\right)=H_{0}^{2}\left(M_{0}, H^{0}\left(I, L_{0}\right)\right), \\
& E_{2,0}^{1,1}=H_{0}^{1}\left(L_{0} / I, H^{1}\left(I, L_{0}\right)\right)=H_{0}^{1}\left(M_{0}, H^{1}\left(I, L_{0}\right)\right), \\
& E_{2,0}^{0,2}=H_{0}^{0}\left(L_{0} / I, H^{2}\left(I, L_{0}\right)\right)=H_{0}^{0}\left(M_{0}, H^{2}\left(I, L_{0}\right)\right) .
\end{aligned}
$$

Очевидно, что $H^{0}\left(I, L_{0}\right)=L_{0}, H^{1}\left(I, L_{0}\right)=L_{0}, H^{2}\left(I, L_{0}\right)=0, H^{1}\left(M_{0}\right)=0$ и, значит,

$$
\begin{gathered}
E_{2,0}^{0,2}=0, \\
E_{2,0}^{1,1}=H_{0}^{1}\left(M_{0}, L_{0}\right)=H_{0}^{1}\left(M_{0}, M_{0}\right) \oplus H_{0}^{1}\left(M_{0}\right)=H_{0}^{1}\left(M_{0}, M_{0}\right), \\
E_{2,0}^{2,0}=H_{0}^{2}\left(M_{0}, L_{0}\right)=H_{0}^{2}\left(M_{0}, M_{0}\right) \oplus H_{0}^{2}\left(M_{0}\right)=H_{0}^{2}\left(M_{0}\right)
\end{gathered}
$$

$H_{0}^{2}\left(M_{0}, M_{0}\right)=0$ по условию.

Пусть $\varphi \in H_{0}^{1}\left(M_{0}, M_{0}\right)=\operatorname{Der}_{0} M_{0} / \operatorname{ad}_{0} M_{0}$, где $\operatorname{Der}_{0}\left(\right.$ соответственно $\left.\operatorname{ad}_{0}\right)-$ пространство дифференцирований (соответственно внутренних дифференцирований) нулевого веса. Тогда $\varphi\left(e_{\alpha}\right)=\widehat{\varphi}\left(e_{\alpha}\right) e_{\alpha}, \widehat{\varphi}\left(e_{\alpha}\right) \in K$, для любого $e_{\alpha} \in M_{0}$. Обозначим через $\mathscr{H}_{0}$ подалгебру Картана алгебры Ли $M_{0}$. Из равенства

$$
0=d \varphi\left(e_{\alpha}, e_{-\alpha}\right)=\left[e_{\alpha}, \varphi\left(e_{-\alpha}\right)\right]-\left[e_{-\alpha}, \varphi\left(e_{\alpha}\right)\right]-\varphi\left(h_{\alpha}\right)
$$


следует, что $\varphi\left(h_{\alpha}\right)=\widehat{\varphi}\left(h_{\alpha}\right) h_{\alpha}, \widehat{\varphi}\left(h_{\alpha}\right) \in K$. Тогда

$$
0=d \varphi\left(h_{\alpha}, e_{\alpha}\right)=\left[h_{\alpha}, \varphi\left(e_{\alpha}\right)\right]-\left[e_{\alpha}, \varphi\left(h_{\alpha}\right)\right]-\varphi\left(\left[h_{\alpha}, e_{\alpha}\right]\right)=-\widehat{\varphi}\left(h_{\alpha}\right)\left[e_{\alpha}, h_{\alpha}\right],
$$

значит, $\varphi\left(h_{\alpha}\right)=\widehat{\varphi}\left(h_{\alpha}\right) h_{\alpha}=0$, когда $h_{\alpha} \in \mathscr{H}_{0}$. Следовательно, $\varphi(h)=0$, если $h \in \mathscr{H}_{0}$. Так как матрица Картана для $M_{0}$ невырождена, то по коэффициентам $\widehat{\varphi}\left(e_{\alpha}\right)$ мы можем найти $h \in \mathscr{H}_{0}$ такой, что $\varphi\left(e_{\alpha}\right)=\widehat{\varphi}\left(e_{\alpha}\right) e_{\alpha}=\operatorname{ad} h\left(e_{\alpha}\right)$ и, следовательно, $\varphi=\operatorname{ad} h$. Таким образом, $E_{2,0}^{1,1}=H_{0}^{1}\left(M_{0}, M_{0}\right)=0$.

Докажем, что $E_{2,0}^{2,0}=H_{0}^{2}\left(M_{0}\right)=0$. Для $\chi \in Z_{0}^{2}\left(M_{0}\right)$

$$
\begin{aligned}
0 & =d \chi\left(e_{\alpha}, e_{-\alpha}, h_{\beta}\right)=-\chi\left(\left[e_{\alpha}, e_{-\alpha}\right], h_{\beta}\right)+\chi\left(\left[e_{\alpha}, h_{\beta}\right], e_{-\alpha}\right)-\chi\left(\left[e_{-\alpha}, h_{\beta}\right], e_{\alpha}\right) \\
& =-\chi\left(h_{\alpha}, h_{\beta}\right)
\end{aligned}
$$

для любых $\alpha, \beta \in R_{0}$. Таким образом, $\chi\left(h_{1}, h_{2}\right)=0$, если $h_{1}, h_{2} \in \mathscr{H}_{0}$.

Присоединенное представление $M_{0}$ эквивалентно сопряженному представлению, следовательно, на $M_{0}$ определено невырожденное инвариантное спаривание $\langle\cdot, \cdot\rangle$. Следуя [10], запишем $\chi \in Z_{0}^{2}\left(M_{0}\right)$ в виде $\chi(x, y)=\langle D x, y\rangle$, где $D$ - линейный оператор на $M_{0},\langle D x, y\rangle=-\langle D y, x\rangle$ (кососимметричность $\chi$ ). Из инвариантности формы $\langle\cdot, \cdot\rangle$ следует, что $D \in \operatorname{Der}_{0}\left(M_{0}\right)$ и $H_{0}^{2}\left(M_{0}\right)=\operatorname{Der}_{0} M_{0} / \operatorname{ad}_{0} M_{0}=$ $H_{0}^{1}\left(M_{0}, M_{0}\right)$. Как показано выше, $H_{0}^{1}\left(M_{0}, M_{0}\right)=0$.

Итак, $\left\{E_{r, 0}^{p, q}\right\}=0$ при $p+q=2$. Следовательно, $H_{0}^{2}\left(L_{0}, L_{0}\right)=0$.

\section{§ 3. Когомологии ненулевого веса}

В этом параграфе мы докажем, что $H^{2}(L, L)=H_{0}^{2}(L, L)$ для всех алгебр Ли $L$, отличных от $C_{2}$ при $p=3$.

ПРЕДЛОЖЕНИЕ 2. 1) Если $R$ - система корней одного из типов $A_{l}, l \geqslant 3$, $D_{l}, E_{6}, E_{7}, E_{8}$, mo $\Lambda_{2}(R)=\{0\}$.

2) Если $\Lambda_{2}(R) \neq\{0\}$, то справедливо одно из следующих утверэсдений:

(a) $R$ - система корней ранга 2 ;

(b) $R$ - система корней одного из типов $B_{l}, C_{l}, l \geqslant 3, F_{4}$. Кроме того, пусть $\left\{\alpha_{1}, \ldots, \alpha_{l}\right\}, l \geqslant 3,-$ базис системь $R,\left\{\alpha_{l-2}, \alpha_{l-1}, \alpha_{l}\right\}-$ базис системы корней $R_{3}$ типа $B_{3}$ или $C_{3}$. Для всех $\alpha, \beta, \gamma \in R$, для которых $\alpha+\beta+\gamma \in \Lambda_{2}(R)$, существует $w \in W(R)$ такой, что $w \alpha, w \beta, w \gamma \in R_{3}$.

ДокАЗАТЕльство. Пусть $\mu \in \Lambda_{2}(R), \mu \neq 0$, тогда $\mu=\alpha+\beta+\gamma, \alpha, \beta, \gamma \in R$, $\alpha \neq \beta$. Рассмотрим векторное подпространство $V_{1}=\langle\alpha, \beta, \gamma\rangle_{\mathbb{R}}$ в $V_{\mathbb{R}}$. Очевидно, что $R_{1}=V_{1} \cap R$ является системой корней в $V_{1}$ ранга не более 3 . Согласно [11; гл. VI, $\S 1$, п. 7, предложение 24] существует базис системы $R$, содержаший базис системы $R_{1}$. Пусть $\left\{\alpha_{1}, \ldots, \alpha_{l}\right\}$ - такой базис. Исследуя строение системы $R_{1}$, мы сможем получить сведения о системе $R$.

Предположим, что $R_{1}$ - приводимая система с базисом $\left\{\delta_{1}, \delta_{2}, \delta_{3}\right\} \subset\left\{\alpha_{1}, \ldots, \alpha_{l}\right\}$ и $\delta_{1}$ не связан с $\delta_{2}, \delta_{3}$, т.е. $\delta_{2}\left(h_{\delta_{1}}\right)=\delta_{3}\left(h_{\delta_{1}}\right)=0$. Один из корней $\alpha, \beta, \gamma$ совпадает с $\delta_{1}$, а два другие - линейные комбинации $\delta_{2}, \delta_{3}$. Следовательно, $\mu=\alpha+$ $\beta+\gamma=\delta_{1}+x \delta_{2}+y \delta_{3}, x, y \in \mathbb{Z}$, и $\mu\left(h_{\delta_{1}}\right)=\delta_{1}\left(h_{\delta_{1}}\right) \not \equiv 0(p)$, что противоречит условию $\mu \in \Lambda_{2}(R) \subset Q_{*}(R)$. 
Аналогично, $R_{1}$ не может являться приводимой системой ранга 2 или системой ранга 1 . Значит, $R_{1}$ - неприводимая система ранга 2 или 3.

Предположим, $R_{1}$ - система типа $A_{3}$. Матрица Картана для системы типа $A_{3}$ невырождена, следовательно, $\Lambda_{2}\left(R_{1}\right) \subset p Q\left(R_{1}\right)$. Однако из вида корней в $A_{3}$ (суммы простых корней) следует, что $\Lambda_{2}\left(R_{1}\right) \cap p Q\left(R_{1}\right)=\{0\}$. Следовательно, $\mu=0$. Полученное противоречие показывает, что $R_{1}$ является системой корней одного из типов $A_{2}, G_{2}, C_{2}, B_{3}, C_{3}$.

Рассмотрим случай, когда $R_{1}$ - система типа $A_{2}$. Пусть $R_{1}$ имеет базис $\left\{\delta_{1}, \delta_{2}\right\} \subset$ $\left\{\alpha_{1}, \ldots, \alpha_{l}\right\}$. Если $R \neq R_{1}$, то сушествует $i$ такой, что $\alpha_{i}$ связан только с одним из простых корней $\delta_{1}, \delta_{2}$, предположим с $\delta_{1}$. Для веса $\mu=\alpha+\beta+\gamma=x \delta_{1}+y \delta_{2}$, $x, y \in \mathbb{Z}$, имеем $\mu\left(h_{\alpha_{i}}\right)=x \delta_{1}\left(h_{\alpha_{i}}\right)$. Так как $\mu \in \Lambda_{2}(R)$, то $x \equiv 0(p)$, следовательно, $y \equiv 0(p)$ и $\mu \in p Q\left(R_{1}\right)$. Но в системе типа $A_{2}$ невозможно набрать такой вес из трех различных корней. Поэтому если $R_{1}$ имеет тип $A_{2}$, то $R$ также имеет тип $A_{2}$. Тем самым доказано 1$)$.

Если $R_{1}$ - система типа $G_{2}$, то $R$ также имеет тип $G_{2}$.

Пусть $R_{1}$ - система корней одного из типов $C_{2}, B_{3}, C_{3}$. Если $R=R_{1}=C_{2}$, то выполняется 2) (а). В остальных случаях система $R$ должна иметь тип $B_{l}, C_{l}$ $(l \geqslant 3)$ или $F_{4}$. Группа $W(R)$ действует транзитивно на множестве базисов $R$. Поэтому найдется $w \in W$ такой, что $w\left(R_{1}\right) \subset R_{3}$. Следовательно, если $R_{1}$ имеет тип $C_{2}$ и $R \neq R_{1}$ или $R_{1}$ имеет тип $B_{3}$ или $C_{3}$, то выполняется 2) (b).

СлЕДСТвИЕ. Если $p>3$, то $\Lambda_{2}(R)=\{0\}$ для любой системъ корней $R$.

ДоКАЗАТЕЛЬСТво. Из предложения следует, что $\Lambda_{2}(R)$ может быть отлично от $\{0\}$ только для систем типа $A_{2}, G_{2}, B_{l}, C_{l}, F_{4}$. При $p>3$ матрица Картана для этих систем невырождена и $\mu \in Q(R)$ принадлежит $\Lambda_{2}(R)$, только если $\mu$ является линейной комбинацией простых корней с коэффициентами, сравнимыми с 0 по модулю $p$. Простая проверка показывает, что при $p>3$ в системах корней $A_{2}, G_{2}, C_{2}$, $B_{3}, C_{3}, F_{4}$ нельзя выбрать три корня, хотя бы два из которых различны, которые в сумме дают ненулевую линейную комбинацию простых корней с коэффициентами, кратными $p$. Таким образом, $\Lambda_{2}(R)=\{0\}$ при $p>3$ для всех систем $R$.

Лемма 2. Пусть $p=3, R$ - система корней одного из типов $B_{l}, C_{l}, F_{4}$, $l \geqslant 3$. Если $\Lambda_{2}(R) \neq\{0\}$, то $\Lambda_{2}(R)=\{3 \delta, \delta-$ короткий корень системиъ $R\} \cup\{0\}$.

ДокаЗАТЕЛЬСтво. Пусть $\mu \in \Lambda_{2}(R), \mu \neq 0$, тогда $\mu=\alpha+\beta+\gamma, \alpha, \beta, \gamma \in R$, $\alpha \neq \beta$. Согласно предложению 2 существует $w \in W$ такое, что $w \alpha, w \beta, w \gamma \in R_{3}$, где $R_{3}$ - система типа $B_{3}$ или $C_{3}$. Заменяя $\mu$ на $w \mu$, будем считать, что $\alpha, \beta, \gamma \in R_{3}$.

Так как матрица Картана для $L$ невырождена, то $\Lambda_{2}(R) \subset p Q(R)$ и $\mu$ можно представить в виде $3 \delta$ для некоторого $\delta \in Q\left(R_{3}\right)$. Если $\left\{\delta_{1}, \delta_{2}, \delta_{3}\right\}$ - базис системы $R_{3}$, то $\mu=x \delta_{1}+y \delta_{2}+z \delta_{3}$, где $x, y, z \equiv 0(3)$.

Так как $\mu=3 \delta \in \Lambda_{2}\left(R_{3}\right)$, то условие $\delta \notin R_{3}$ влечет, что $\mu$ не пропорционален никакому корню из $R_{3}$. Следовательно, можно считать, что коэффициент $x$ положительный, а один из $y, z$ (например, $y$ ) отрицательный (см. [12; гл. VIII, $\S 4$, п. 3 , лемма 10]). Абсолютная величина коэффициентов в разложении корней системы $R_{3}$ по базису не превосходит 2 . Для того чтобы $x>0$ делился на 3 , хотя бы два 
из корней $\alpha, \beta, \gamma$ должны быть положительными, а для того чтобы $y<0$ делился на 3 , хотя бы два из корней $\alpha, \beta, \gamma$ должны быть отрицательными. Получили противоречие. Следовательно, $\delta$ является корнем системы $R_{3}$.

Предположим, что $\delta$ - длинный корень. Так как максимальный корень $\gamma_{0}$ системы $R$ является длинным корнем и $W$ действует транзитивно на множестве корней одинаковой длины, то $3 \gamma_{0} \in \Lambda_{2}(R)$. Очевидно, $3 \gamma_{0}$ только одним способом представляется в виде суммы трех корней: $3 \gamma_{0}=\gamma_{0}+\gamma_{0}+\gamma_{0}$. Поэтому $3 \gamma_{0}$ не является весом $C^{2}(L, L)$ и, следовательно, $3 \gamma_{0} \notin \Lambda_{2}(R)$. Полученное противоречие показывает, что $\delta$ - короткий корень.

ПРЕДЛОЖЕНИЕ $3 . H^{2}(L, L)=H_{0}^{2}(L, L)$ для классической алгебры Ли $L$ над полем характеристики $p>2$, отличной от алгебры Ли типа $C_{2}$ при $p=3$.

ДоКАЗАТЕЛЬСТво. Согласно предложению 2 и следствию из него остается доказать утверждение только при $p=3$ для алгебр типов $B_{l}, C_{l}, l \geqslant 3, F_{4}, A_{2}$, $\bar{A}_{2}, A_{2}^{z}$.

Пусть $L$ - алгебра Ли одного из типов $B_{l}, C_{l}, l \geqslant 3, F_{4}$. Из леммы 2 следует, что если $\Lambda_{2}(R) \neq\{0\}$, то ненулевые веса из $\Lambda_{2}(R)$ имеют вид $3 \delta$, где $\delta$ - короткий корень из $R$, и, следовательно, группа $W$ действует транзитивно на множестве ненулевых элементов $\Lambda_{2}(R)$. Таким образом, группы $H_{\mu_{1}}^{2}(L, L)$ и $H_{\mu_{2}}^{2}(L, L)$ изоморфны для любых ненулевых $\mu_{1}, \mu_{2}$.

Покажем, что $H_{\mu}^{2}(L, L)=Z_{\mu}^{2}(L, L)$ для $\mu=3 \delta \in \Lambda_{2}(R)$. Каждый вес $C^{1}(L, L)$ является суммой двух корней. Пусть $\alpha, \beta \in R$ и $\alpha+\beta=3 \delta$. Так как $p=3$, то $\left.\alpha\right|_{\mathscr{H}}=-\left.\beta\right|_{\mathscr{H}}$. Согласно [13; $\S 4$, предложение 4.2$]$ корневые пространства относительно $\mathscr{H}$ в $L$ одномерны, т.е. все корни как функции на $\mathscr{H}$ различны. Следовательно, $\alpha=-\beta$ и $\alpha+\beta=0$ в $R$. Таким образом, ни для каких $\alpha, \beta \in R$ не выполняется равенство $\alpha+\beta=3 \delta$. Поэтому $C_{3 \delta}^{1}(L, L)=0$ и $H_{\mu}^{2}(L, L)=Z_{\mu}^{2}(L, L)$ для $\mu \in \Lambda_{2}(R), \mu \neq 0$.

Пусть $\psi \in Z_{3 \delta}^{2}(L, L), \psi \neq 0$. Сушествуют $\alpha, \beta, \gamma \in R$ такие, что $\psi\left(e_{\alpha}, e_{\beta}\right)=$ $x e_{\gamma}, x \neq 0$. Из предложения 2 следует, что существует $w \in W$ такое, что $w \alpha, w \beta, w \gamma \in R_{3}$. Тогда $\varphi=\left.w \psi\right|_{L_{3}} \neq 0$, где $L_{3}$ - подалгебра в $L$ с системой корней $R_{3}$. Так как $\varphi$ имеет вес $3 w \delta$, то $\varphi$ переводит $L_{3} \times L_{3}$ в $L_{3}$ и, следовательно, $\varphi \in Z_{3 w \delta}^{2}\left(L_{3}, L_{3}\right)$. Форма Киллинга для алгебр типа $B_{3}$ или $C_{3}$ невырождена над полем характеристики 3 , что следует из формулы (1). Это дает тривиальность $H^{2}\left(L_{3}, L_{3}\right)$ для $L_{3}$ типа $B_{3}$ и $C_{3}$, в частности, $H_{3 \delta}^{2}\left(L_{3}, L_{3}\right)=Z_{3 \delta}^{2}\left(L_{3}, L_{3}\right)=0$. Полученное противоречие доказывает, что $\psi=0$ и $H_{3 \delta}^{2}(L, L)=0$ для любого короткого корня $\delta$.

Пусть $L$ - алгебра Ли типа $A_{2}, \bar{A}_{2}$ или $A_{2}^{z}, p=3,\{\alpha, \beta\}$ - базис системы корней $R$. Матрища Картана для $R$ при $p=3$ вырождена. Нетрудно показать, что если $\Lambda_{2}(R) \neq\{0\}$, то $\Lambda_{2}(R)=\{0, \pm(\alpha+2 \beta), \pm(\alpha-\beta), \pm(2 \alpha+\beta)\}$. Так как $\alpha-\beta=w_{\beta}(\alpha+2 \beta),-2 \alpha-\beta=w_{\alpha}(\alpha-\beta)$, то все ненулевые элементы в $\Lambda_{2}(R)$ сопряжены относительно групшы Вейля и, следовательно, достаточно доказать, что $H_{\alpha+2 \beta}^{2}(L, L)=0$. Если $L$ имеет тип $A_{2}^{z}$, то базисные векторы $C_{\alpha+2 \beta}^{2}(L, L)$ являются собственньми для $z$ с ненулевыми собственными значениями. Так как $L$ действует тривиально на $H^{2}(L, L)$, то $H_{\alpha+2 \beta}^{2}(L, L)=0$. Если $L$ имеет тип $A_{2}, \bar{A}_{2}$, то тривиальность $H_{\alpha+2 \beta}^{2}(L, L)$ проверяется непосредственным вычислением. 
Пространство $C_{\alpha+2 \beta}^{1}(L, L)$ двумерно и порождается векторами $b_{1}=e_{-\alpha-\beta}^{*} \otimes e_{\beta}$ и $b_{2}=e_{-\beta}^{*} \otimes e_{\alpha+\beta}$.

Имеем

$$
\begin{aligned}
& d b_{1}\left(e_{-\beta}, e_{-\alpha-\beta}\right)=\left[e_{-\beta}, b_{1}\left(e_{-\alpha-\beta}\right)\right]=\left[e_{-\beta}, e_{\beta}\right]=-h_{\beta} \\
& d b_{2}\left(e_{-\beta}, e_{-\alpha-\beta}\right)=-\left[e_{-\alpha-\beta}, b_{2}\left(e_{-\beta}\right)\right]=-\left[e_{-\alpha-\beta}, e_{\alpha+\beta}\right]=h_{\alpha}+h_{\beta}
\end{aligned}
$$

Таким образом, если $L$ имеет тип $A_{2}$, то $d b_{1}, d b_{2}$ линейно независимы и

$$
\operatorname{dim} B_{\alpha+2 \beta}^{2}(L, L)=2
$$

Если $L$ имеет тип $\bar{A}_{2}$, то

$$
1 \leqslant \operatorname{dim} B_{\alpha+2 \beta}^{2}(L, L) \leqslant 2 .
$$

Пусть $L$ имеет тип $A_{2}$. Пространство $C_{\alpha+2 \beta}^{2}(L, L)$ имеет размерность 8 и порождается векторами

$$
\begin{array}{ll}
c_{1}=h_{\alpha}^{*} \wedge e_{-\alpha-\beta}^{*} \otimes e_{\beta}, & c_{2}=h_{\alpha}^{*} \wedge e_{-\beta}^{*} \otimes e_{\alpha+\beta}, \\
c_{3}=h_{\beta}^{*} \wedge e_{-\alpha-\beta}^{*} \otimes e_{\beta}, & c_{4}=h_{\beta}^{*} \wedge e_{-\beta}^{*} \otimes e_{\alpha+\beta}, \\
\psi_{1}=e_{-\alpha-\beta}^{*} \wedge e_{\alpha}^{*} \otimes e_{\alpha+\beta}, & \psi_{2}=e_{-\beta}^{*} \wedge e_{-\alpha}^{*} \otimes e_{\beta}, \\
\psi_{3}=e_{-\alpha-\beta}^{*} \wedge e_{-\beta}^{*} \otimes h_{\alpha}, & \psi_{4}=e_{-\alpha-\beta}^{*} \wedge e_{-\beta}^{*} \otimes h_{\beta} .
\end{array}
$$

Пусть $\psi \in Z_{\alpha+2 \beta}^{2}(L, L)$,

$$
\psi=a_{1} c_{1}+a_{2} c_{2}+a_{3} c_{3}+a_{4} c_{4}+x_{1} \psi_{1}+x_{2} \psi_{2}+x_{3} \psi_{3}+x_{4} \psi_{4} .
$$

Тогда

$$
\begin{aligned}
0= & d \psi\left(h, e_{-\alpha-\beta}, e_{-\beta}\right)=-\left[e_{-\alpha-\beta}, \psi\left(h, e_{-\beta}\right)\right]+\left[e_{-\beta}, \psi\left(h, e_{-\alpha-\beta}\right)\right] \\
& -\psi\left(\left[h, e_{-\alpha-\beta}\right], e_{-\beta}\right)+\psi\left(\left[h, e_{-\beta}\right], e_{-\alpha-\beta}\right)=-\left[e_{-\alpha-\beta}, \psi\left(h, e_{-\beta}\right)\right] \\
& +\left[e_{-\beta}, \psi\left(h, e_{-\alpha-\beta}\right)\right]+(\alpha+2 \beta)(h) \psi\left(e_{-\alpha-\beta}, e_{-\beta}\right) \\
= & -\left[e_{-\alpha-\beta}, \psi\left(h, e_{-\beta}\right)\right]+\left[e_{-\beta}, \psi\left(h, e_{-\alpha-\beta}\right)\right] .
\end{aligned}
$$

При $h=h_{\alpha}$ получим

$$
0=a_{2}\left(h_{\alpha}+h_{\beta}\right)-a_{1} h_{\beta}=a_{2} h_{\alpha}+\left(a_{2}-a_{1}\right) h_{\beta} .
$$

Если $h=h_{\beta}$, то

$$
0=a_{4} h_{\alpha}+\left(a_{4}-a_{3}\right) h_{\beta}
$$

Таким образом, $a_{1}=a_{2}=a_{3}=a_{4}=0$.

Покажем, что размерность $Z_{\alpha+2 \beta}^{2}(L, L)$ не превышает 2 .

$$
\begin{aligned}
0 & =d \psi\left(e_{-\alpha-\beta}, e_{\alpha}, e_{-\beta}\right)=\left[e_{-\beta}, \psi\left(e_{-\alpha-\beta}, e_{\alpha}\right)\right]-\left[e_{\alpha}, \psi\left(e_{-\alpha-\beta}, e_{-\beta}\right)\right] \\
& =\left[e_{-\beta}, x_{1} e_{\alpha+\beta}\right]-\left[e_{\alpha}, x_{3} h_{\alpha}+x_{4} h_{\beta}\right]=\left(N x_{1}+2 x_{3}-x_{4}\right) e_{\alpha}
\end{aligned}
$$


где $\left[e_{-\beta}, e_{\alpha+\beta}\right]=N e_{\alpha}, N \in K^{*}$. Аналогично,

$$
0=d \psi\left(e_{-\beta}, e_{-\alpha}, e_{-\alpha-\beta}\right)=\left(M x_{2}+2 x_{3}-x_{4}\right) e_{-\alpha},
$$

где $\left[e_{-\alpha-\beta}, e_{\beta}\right]=M e_{-\alpha}, M \in K^{*}$.

Мы получили два линейно независимых соотношения: $N x_{1}+2 x_{3}-x_{4}=0$, $M x_{2}+2 x_{3}-x_{4}=0$. Следовательно,

$$
\operatorname{dim} Z_{\alpha+2 \beta}^{2}(L, L) \leqslant 2 .
$$

Так как $\operatorname{dim} B_{\alpha+2 \beta}^{2}(L, L)=2$, то $H_{\alpha+2 \beta}^{2}(L, L)=0$.

Пусть $L$ имеет тип $\bar{A}_{2}$. В этом случае $h_{\alpha}=h_{\beta}, h_{\alpha+\beta}=-h_{\alpha}$. Пространство $C_{\alpha+2 \beta}^{2}(L, L)$ имеет размерность 5 и порождается векторами $c_{1}, c_{2}, \psi_{1}, \psi_{2}, \psi_{3}$. Пусть $\psi \in Z_{\alpha+2 \beta}^{2}(L, L), \psi=a_{1} c_{1}+a_{2} c_{2}+x_{1} \psi_{1}+x_{2} \psi_{2}+x_{3} \psi_{3}$. Из (2) при $h=h_{\alpha_{1}}$ получим

$$
0=a_{2}\left(-h_{\alpha}\right)-a_{1} h_{\alpha}=\left(-a_{2}-a_{1}\right) h_{\alpha} .
$$

Из (3), (4) при $x_{4}=0$ получим

$$
\begin{aligned}
\left(N x_{1}+2 x_{3}\right) e_{\alpha} & =0 \\
\left(M x_{2}+2 x_{3}\right) e_{-\alpha} & =0
\end{aligned}
$$

$N, M \in K^{*}$. Имеем

$$
\begin{aligned}
0 & =d \psi\left(e_{\alpha}, e_{-\alpha}, e_{-\alpha-\beta}\right) \\
& =-\left[e_{-\alpha}, \psi\left(e_{\alpha}, e_{-\alpha-\beta}\right)\right]-\psi\left(\left[e_{\alpha}, e_{-\alpha}\right], e_{-\alpha-\beta}\right)+\psi\left(\left[e_{\alpha}, e_{-\alpha-\beta}\right], e_{-\alpha}\right) \\
& =\left[e_{-\alpha}, x_{1} e_{\alpha+\beta}\right]-\psi\left(h_{\alpha}, e_{-\alpha-\beta}\right)+\psi\left(N_{2} e_{-\beta}, e_{-\alpha}\right) \\
& =\left(N_{1} x_{1}-a_{1}+N_{2} x_{2}\right) e_{\beta},
\end{aligned}
$$

где $\left[e_{-\alpha}, e_{\alpha+\beta}\right]=N_{1} e_{\beta},\left[e_{\alpha}, e_{-\alpha-\beta}\right]=N_{2} e_{-\beta}, N_{1}, N_{2} \in K^{*}$.

Получили 4 линейно независимых соотношения:

$$
-a_{1}-a_{2}=0, \quad N x_{1}+2 x_{3}=0, \quad M x_{2}+2 x_{3}=0, \quad N_{1} x_{1}-a_{1}+N_{2} x_{2}=0 .
$$

Следовательно, $\operatorname{dim} Z_{\alpha+2 \beta}^{2}(L, L) \leqslant 1$. Так как $1 \leqslant \operatorname{dim} B_{\alpha+2 \beta}^{2}(L, L) \leqslant 2$, то $H_{\alpha+2 \beta}^{2}(L, L)=0$.

\section{§4. Вычисление $H_{0}^{2}(L, L)$}

В этом параграфе для классической алгебры Ли $L$ при $p>2$ индукцией по рангу доказывается тривиальность групшы $H_{0}^{2}(L, L)$ (при $p=3 L$ отлична от $C_{2}$ и $G_{2}$ ). Напомним, что в силу предложения 1 мы можем рассматривать только алгебры Ли с вырожденной формой Киллинга.

Предложение 3 позволяет нам считать, что $H^{2}(L, L)=H_{0}^{2}(L, L)$. Если характеристика поля равна 3 , то индукцию будем начинать с алгебр Ли $L$ типов $A_{1}$, $B_{3}, C_{3}$; если $p>3$, то индукция начинается с $A_{1}, B_{2}$. Из формулы (1) следует, что 
форма Киллинга для этих алгебр невырождена, и из предложения 1 следует, что $H_{0}^{2}(L, L)=0$.

Пусть $L$ - алгебра Ли ранга $l$. Предположим, что $H_{0}^{2}(L, L)=0$ для алгебр $L$ ранга меньше $l$. Выберем коцикл $\psi \in Z_{0}^{2}(L, L)$. Обозначим $L \otimes K[\varepsilon]$, где $K[\varepsilon]-$ алгебра двойных чисел, через $L(\varepsilon)$ и отождествим пространство $L$ с $L \otimes 1$. На $L(\varepsilon)$ можно определить структуру алгебры Ли $(L(\varepsilon),[\cdot, \cdot])$, если продолжить лиевское умножение с $L$. При помощи коцикла $\psi$ определим на $L(\varepsilon)$ новую структуру алгебры Ли, полагая $x \circ y=[x, y]+\varepsilon \psi(x, y), x, y \in L$. Очевидно, коцикл $\psi$ когомологичен нулю тогда и только тогда, когда алгебры Ли $(L(\varepsilon),[\cdot, \cdot])$ и $(L(\varepsilon), \circ)$ изоморфны. В дальнейшем через $L(\varepsilon)$ будем обозначать алгебру Ли $(L(\varepsilon), \circ)$.

Так как коцикл $\psi$ имеет нулевой вес, то для корневых векторов $e_{\alpha}, e_{\beta}$

$$
e_{\alpha} \circ e_{\beta} \equiv\left[e_{\alpha}, e_{\beta}\right] \quad\left(\bmod \varepsilon L_{\alpha+\beta}\right), \quad h \circ e_{\alpha} \equiv\left[h, e_{\alpha}\right] \quad\left(\bmod \varepsilon L_{\alpha}\right)
$$

В частности, если $\alpha+\beta \notin R$, то $e_{\alpha} \circ e_{\beta}=0$.

Для $\gamma \in R$ положим $L_{\gamma}(\varepsilon)=L_{\gamma} \otimes K[\varepsilon] ; L_{\gamma}(\varepsilon)$ имеет базис $\left\{e_{\gamma}, \varepsilon e_{\gamma}\right\}$ над $K$. Из $(5)$ следует, что в $L(\varepsilon)$ можно построить градуировку по системе корней, как это сделано для $L$ в $\S 2$ :

$$
L(\varepsilon)=L_{-2}(\varepsilon)+L_{-1}(\varepsilon)+L_{0}(\varepsilon)+L_{1}(\varepsilon)+L_{2}(\varepsilon)
$$

где $L_{0}(\varepsilon)=(\mathscr{H} \otimes K[\varepsilon]) \oplus \bigoplus_{\gamma \in R_{0}} L_{\gamma}(\varepsilon), L_{i}(\varepsilon)=\bigoplus_{\gamma \in R_{i}} L_{\gamma}(\varepsilon), i \in\{-2,-1,1,2\}$, $R_{i}$ - подмножества системы $R$ (см. табл. 1$)$.

Согласно лемме 1 по предположению индукции можно считать, что $\left.\psi\right|_{L_{0}}=0$ и алгебра Ли $L_{0} \otimes K[\varepsilon]$ с умножением $[\cdot, \cdot]$ изоморфна $L_{0}(\varepsilon)$ с умножением о. Подалгебру $L_{0} \otimes 1$ отождествим с $L_{0}$. Напомним, что $L_{0}=M_{0} \oplus\left\langle h_{0}\right\rangle$, где $M_{0}$ - алгебра Ли классического типа ранга $l-1,\left\langle h_{0}\right\rangle$ - центр $L_{0}$ (во всех случаях, когда форма Киллинга на $L$ вырождена, центр в $L_{0}$ отшепляется, см. табл. 1$), \operatorname{dim} L_{ \pm 2} \leqslant 1$.

Пусть $\mathscr{H}=\mathscr{H}_{0}+\left\langle h_{0}\right\rangle$, где $\mathscr{H}_{0}-$ подалгебра Картана в $M_{0}$.

ПРЕДЛОЖЕНИЕ 4. 1) $L_{-1}(\varepsilon)$ является ограниченным $M_{0}$-модулем.

2) $\psi\left(h, L_{-1}\right)=0$ для любого $h \in \mathscr{H}_{0}$.

ДокАзАТЕльство. Обозначим через $\Sigma$ множество всех корней $\alpha \in R_{0}$, для которых сушествует $\gamma \in R_{-1}$ такой, что $\alpha$-серия, содержашая $\gamma$, имеет длину 1. Пусть $L(\alpha)=\left\langle e_{\alpha}, e_{-\alpha}, h_{\alpha}\right\rangle$. Очевидно, если $\alpha \in \Sigma$, то в $L_{-1}$ содержится двумерньй $L(\alpha)$-подмодуль.

Для доказательства предложения 4 понадобится следующая лемма.

Лемма 3. $\mathscr{H}_{0}=\left\langle h_{\alpha}, \alpha \in \Sigma\right\rangle$.

ДокаЗАтельство. Пусть $\left\{\alpha_{1}, \ldots, \alpha_{l}\right\}-$ базис $R$. Длина $\alpha$-серии, содержащей $\beta$, равна 1 для неортогональных корней $\alpha, \beta$ одинаковой длины. Поэтому $\alpha \in \Sigma$, если существует корень $\beta \in R_{-1}$ такой, что $(\alpha, \beta) \neq 0$ и $\|\alpha\|=\|\beta\|$. Если такой корень $\beta$ для $\alpha$ сушествует, обозначим его через $\widehat{\alpha}$.

Пусть $\gamma_{1}, \gamma_{2}-$ корни одинаковой длины из $R_{0}$. Для некоторого $w_{0} \in W\left(R_{0}\right)$ выполняется $w_{0} \gamma_{1}=\gamma_{2}$. Для любого корня $\gamma \in R_{-1} w_{0} \gamma$ снова принадлежит $R_{-1}$. Следовательно, если для $\gamma_{1}$ сушествует $\widehat{\gamma}_{1}$, то и для $\gamma_{2}$ сушествует $\widehat{\gamma}_{2}$, так как $w_{0}$ 
сохраняет скалярное произведение. Таким образом, $\Sigma$ инвариантно относительно $W\left(R_{0}\right)$.

Все корни в системах $A_{l}, D_{l}, E_{6}, E_{7}, E_{8}$ имеют одинаковую длину, и, следовательно, для всех корней $\alpha \in R_{0}$ сушествует $\widehat{\alpha}$ и $\Sigma=R_{0}$.

Если $L$ - алгебра типа $B_{l}, l \geqslant 3$, то $R_{0}$ - система типа $B_{l-1}$ с базисом $\left\{\alpha_{2}, \ldots, \alpha_{l}\right\}$, где $\alpha_{2}, \ldots, \alpha_{l-1}-$ длинные корни, $\alpha_{l}-$ короткий корень. Для $\alpha_{2}$ сушествует $\widehat{\alpha}_{2}=$ $-\alpha_{1}$, следовательно, для любого длинного корня $\alpha$ сушествует $\widehat{\alpha}$. Таким образом, $\Sigma$ содержит все длинные корни. Покажем, что элементы $h_{\gamma}, \gamma$ - длинный корень, порождают $\mathscr{H}_{0}$. Любой элемент $h_{\gamma} \in \mathscr{H}_{0}$ раскладывается по $h_{\alpha_{i}}, \alpha_{i}-$ простой корень. Покажем, что $h_{\alpha_{l}}$ раскладьвается в линейную комбинацию $h_{\gamma}, \gamma-$ длинньй корень.

Число Картана $\left\langle\alpha_{l}, \alpha_{l-1}\right\rangle=-1$, следовательно, $h_{w_{\alpha_{l}} \alpha_{l-1}}=w_{\alpha_{l}} h_{\alpha_{l-1}}=$ $h_{\alpha_{l-1}}-\left\langle\alpha_{l}, \alpha_{l-1}\right\rangle h_{\alpha_{l}}=h_{\alpha_{l-1}}+h_{\alpha_{l}}$ и $h_{\alpha_{l}}=h_{w_{\alpha_{l}} \alpha_{l-1}}-h_{\alpha_{l-1}}$. Так как $\alpha_{l-1}-$ длинный корень, то $w_{\alpha_{l}} \alpha_{l-1}-$ длинный корень.

Пусть $L$ - алгебра типа $C_{l}$, и пусть $R$ имеет базис $\left\{\alpha_{1}, \ldots, \alpha_{l}\right\}, R_{0}$ имеет базис $\left\{\alpha_{1}, \ldots, \alpha_{l-1}\right\}, R_{0}$ - система типа $A_{l}$ и все корни в $R_{0}$ короткие. Для $\alpha_{1}$ существует $\widehat{\alpha}_{1}=-\alpha_{2}-\cdots-\alpha_{l}$, следовательно, для всех $\alpha_{i} \in R_{0}$ сушествует $\widehat{\alpha}_{i}$ и $\Sigma=R_{0}$.

Пусть $R$ - система корней типа $F_{4}$ с базисом $\left\{\alpha_{1}, \alpha_{2}, \alpha_{3}, \alpha_{4}\right\}$, где $\alpha_{1}, \alpha_{2}-$ длинные корни, $\alpha_{3}, \alpha_{4}-$ короткие корни, $R_{0}$ имеет базис $\left\{\alpha_{2}, \alpha_{3}, \alpha_{4}\right\}$. Легко найти $\widehat{\alpha}_{2}, \widehat{\alpha}_{4}: \widehat{\alpha}_{2}=-\alpha_{1}$ и $\widehat{\alpha}_{4}=-\alpha_{1}-\alpha_{2}-\alpha_{3} ; \widehat{\alpha}_{3}$ сушествует, так как $\left\|\alpha_{3}\right\|=\left\|\alpha_{4}\right\|$. Следовательно, $\Sigma=R_{0}$.

Продолжим доказательство предложения 4. Пусть $V=L_{-1}(\varepsilon)$, обозначим через $V_{\gamma}$ векторное пространство над $K$, натянутое на $e_{\gamma}, \varepsilon e_{\gamma}$. Положим $\operatorname{ad}_{\varepsilon} m(v)=$ $m \circ v, m \in M_{0}, v \in V$. Очевидно, что $\operatorname{ad}_{\varepsilon}^{p} e_{\alpha}=\operatorname{ad}_{\varepsilon}^{p} e_{-\alpha}=0$ для любого $\alpha \in R_{0}$. Для $v \in V_{\lambda}, h_{\alpha} \in \mathscr{H}_{0}$ можно записать $\operatorname{ad}_{\varepsilon} h_{\alpha}(v)=\left(\lambda\left(h_{\alpha}\right)+\varepsilon \hat{\psi}\left(h_{\alpha}, v\right)\right) v$, де $\widehat{\psi}(h, v)$ из $K, \lambda\left(h_{\alpha}\right) \in \mathbb{F}_{p}$. Здесь

$$
\varepsilon \widehat{\psi}\left(h_{\alpha}, v\right) v=x \varepsilon \psi\left(h_{\alpha}, e_{\lambda}\right)
$$

где $v=x e_{\lambda}+y \varepsilon e_{\lambda}, x, y \in K$. Тогда $\operatorname{ad}_{\varepsilon}^{p} h_{\alpha}(v)=\left(\lambda\left(h_{\alpha}\right)+\varepsilon \widehat{\psi}\left(h_{\alpha}, v\right)\right)^{p} v=$ $\lambda^{p}\left(h_{\alpha}\right) v=\lambda\left(h_{\alpha}\right) v$. Если положить $f_{h}(v)=\left(\operatorname{ad}_{\varepsilon}^{p} h-\operatorname{ad}_{\varepsilon} h\right)(v)$, то $f_{h_{\alpha}}(v)=$ $-\varepsilon \widehat{\psi}\left(h_{\alpha}, v\right) v$. Отображение $f_{h_{\alpha}}$ переводит $V$ в $\varepsilon V$ и на $\varepsilon V$ действует нулевьгм образом, значит, $f_{h_{\alpha}}$ можно рассматривать как отображение из $V / \varepsilon V$ в $\varepsilon V$. Поскольку $V / \varepsilon V \cong \varepsilon V$ (изоморфизм неприводимых $M_{0}$-модулей) и $f_{h_{\alpha}}$ перестановочен с действием $M_{0}$, то по лемме Шура $f_{h_{\alpha}}$ действует умножением на скаляр и $\widehat{\psi}\left(h_{\alpha}, v\right)$ не зависит от $v$.

Покажем, что если $\alpha \in \Sigma$, то $f_{h_{\alpha}}=0$. Пусть $U=\left\langle e_{\gamma}, e_{\gamma+\alpha}\right\rangle-$ соответствующий двумерный $L(\alpha)$-подмодуль в $L_{-1}$ в алгебре $L ; U(\varepsilon)=U \otimes K[\varepsilon]$ является $L(\alpha)$-подмодулем в $V$ в алгебре $(L(\varepsilon)$, о ) и имеет композиционный ряд $U(\varepsilon) \supset \varepsilon U(\varepsilon) \supset 0$, факторы которого изоморфны $U$. Кроме того, $U(\varepsilon)$ как модуль над $L(\alpha)$ расщепляется. Действительно,

$$
\operatorname{Ext}^{1}(U, U)=H^{1}\left(L(\alpha), U^{*} \otimes U\right)=H^{1}(\operatorname{sl}(2), \operatorname{gl}(2))=0
$$

Таким образом, $U(\varepsilon) \cong U \oplus \varepsilon U$ и $\widehat{\psi}\left(h_{\alpha},-\right)=0$ на $U(\varepsilon)$, значит, $f_{h_{\alpha}}=\operatorname{ad}_{\varepsilon}^{p} h_{\alpha}-$ $\operatorname{ad}_{\varepsilon} h_{\alpha}=0$. Из леммы 3 следует, что $f_{h}=0$ для любого $h \in \mathscr{H}_{0}$. Тем самым доказано 1). 
Докажем 2). Так как $\widehat{\psi}\left(h_{\alpha},-\right)=0$ для любого $\alpha \in \Sigma$, то из (6) получаем, что $\psi\left(h_{\alpha}, L_{-1}\right)=0$ для $\alpha \in \Sigma$. Из леммы 3 следует, что $\psi\left(h, L_{-1}\right)=0$ для любого $h \in \mathscr{H}_{0}$.

ПРЕДЛОЖЕНИЕ 5. $M_{0}$-модуль $L_{-1}(\varepsilon)$ раскладъвается в прямую сумму подмодулей, изоморфных $L_{-1}, L_{-1}(\varepsilon)=L_{-1}^{\prime}+\varepsilon L_{-1}^{\prime}$.

ДокАЗАТЕЛЬство. Пусть $V=L_{-1}(\varepsilon)$. Обозначим через $u\left(M_{0}\right) \quad u$-алгебру алгебры Ли $M_{0}, u\left(M_{0}\right)=u\left(M_{0}^{-}\right) u\left(\mathscr{H}_{0}\right) u\left(M_{0}^{+}\right)$, где $M_{0}=M_{0}^{-}+\mathscr{H}_{0}+M_{0}^{+}-$ треугольное разложение алгебры $M_{0}$. Пусть $\mu \in R_{-1}$ - такой корень, что $\mu+\alpha \notin$ $R_{-1}$ для любого $\alpha \in R_{0}^{+}$. Очевидно, $\mu$ - старший вес $L_{0}$-модуля $L_{-1}$. Пусть $v_{\mu} \in L_{\mu}(\varepsilon), v_{\mu} \notin \varepsilon V$. Тогда $u\left(M_{0}^{+}\right) v_{\mu}=0, u\left(\mathscr{H}_{0}\right) v_{\mu}=\left\langle v_{\mu}\right\rangle$ (предложение 3 ). Отсюда получаем, что $u\left(M_{0}\right) v_{\mu}=u\left(M_{0}^{-}\right) v_{\mu}$. Покажем, что $V=u\left(M_{0}\right) v_{\mu} \oplus$ $\varepsilon u\left(M_{0}\right) v_{\mu}$ - прямая сумма $M_{0}$-модулей.

Пусть $\pi: V \rightarrow V / \varepsilon V$ каноническая проекция, $\pi\left(v_{\mu}\right)=\bar{v}_{\mu} \neq 0$. Так как $V / \varepsilon V$ - неприводимый $M_{0}$-модуль, то $\pi\left(u\left(M_{0}\right) v_{\mu}\right)=u\left(M_{0}\right) \bar{v}_{\mu}=V / \varepsilon V$. Предположим, что $A=u\left(M_{0}\right) v_{\mu} \cap \varepsilon V \neq 0, A-$ подмодуль в $\varepsilon V$. Так как $\varepsilon V-$ неприводимый

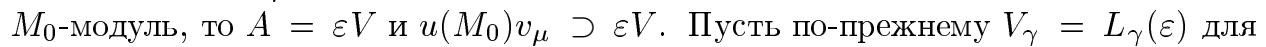
$\gamma \in R_{-1}$. Так как $u\left(M_{0}\right) v_{\mu}=u\left(M_{0}^{-}\right) v_{\mu}$, то $u\left(M_{0}\right) v_{\mu} \subset\left\langle v_{\mu}\right\rangle \oplus \bigoplus_{\gamma \neq \mu} V_{\gamma}$.

Следовательно, $u\left(M_{0}\right) v_{\mu} \cap V_{\mu}=\left\langle v_{\mu}\right\rangle$. С другой стороны, $\left\langle\varepsilon v_{\mu}\right\rangle=\varepsilon V \cap V_{\mu} \subset$ $u\left(M_{0}\right) v_{\mu} \cap V_{\mu}=\left\langle v_{\mu}\right\rangle$. Полученное противоречие доказьвает, что $A=0$ и $V=$ $u\left(M_{0}\right) v_{\mu} \oplus \varepsilon u\left(M_{0}\right) v_{\mu}$.

Согласно предложению 5 можно считать, что $\psi\left(v_{1}, v_{0}\right)=0$, если $v_{1} \in L_{-1}$,

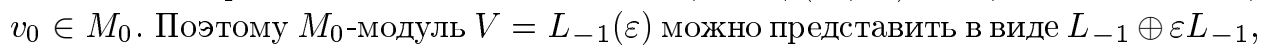
где $L_{-1}=L_{-1} \otimes 1$. Аналогично, для $L_{1}(\varepsilon)$ получаем разложение в прямую сумму изоморфных неприводимых $M_{0}$-модулей: $L_{1}(\varepsilon)=L_{1} \oplus \varepsilon L_{1}$ (т.е. можно считать, что $\left.\psi\left(L_{1}, M_{0}\right)=0\right)$.

Дальнейшее доказательство тривиальности коцикла $\psi$ будет различным для случаев, когда $L$ не имеет центра и когда $L$ имеет центр.

Предположим, что алгебра Ли $L$ не имеет центра.

Если $L_{-2} \neq 0$, то $\left[L_{-1}, L_{-1}\right]=L_{-2}=\left\langle e_{\gamma}\right\rangle$. Так как $L_{-2}$ одномерно, то $\psi\left(e_{\alpha}, e_{\gamma}\right)=0$ для любого $\alpha \in R_{0}$. Отсюда, учитьвая, что $\psi\left(L_{0}, L_{0}\right)=0$, получаем $0=d \psi\left(e_{\alpha}, e_{-\alpha}, e_{\gamma}\right)=-\psi\left(\left[e_{\alpha}, e_{-\alpha}\right], e_{\gamma}\right)=-\psi\left(h_{\alpha}, e_{\gamma}\right)$. Следовательно, $\psi\left(L_{-2}, M_{0}\right)=0$. Аналогично, $\psi\left(L_{2}, M_{0}\right)=0$.

Для $v_{1}, v_{2} \in L_{-1}$ положим $\left[v_{1}, v_{2}\right]=\left\langle v_{1}, v_{2}\right\rangle_{1} e_{\gamma}$, где $\langle\cdot, \cdot\rangle_{1}-M_{0}$-инвариантная невырожденная кососимметрическая форма на $L_{-1}$. В $L(\varepsilon) v_{1} \circ v_{2} \in L_{-2}(\varepsilon)$ и $v_{1} \circ v_{2}=\left\langle v_{1}, v_{2}\right\rangle_{1} e_{\gamma}+\varepsilon\left\langle v_{1}, v_{2}\right\rangle_{2} e_{\gamma}$, где $\langle\cdot, \cdot\rangle_{2}$ - также $M_{0}$-инвариантная невырожденная кососимметрическая форма на $L_{-1}$. Так как $L_{-1}-$ неприводимый

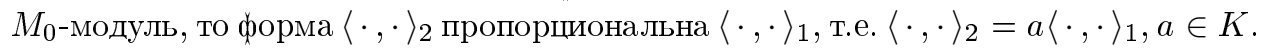
Заменяя $e_{\gamma}$ на $e_{\gamma}+\varepsilon a e_{\gamma}$, получаем $v_{1} \circ v_{2}=\left\langle v_{1}, v_{2}\right\rangle_{1} e_{\gamma}$. Таким образом, можно считать, что $\psi\left(L_{-1}, L_{-1}\right)=0$. Аналогично, подправив базис в $L_{2}(\varepsilon)$, можно считать, что $\psi\left(L_{1}, L_{1}\right)=0$.

Выясним, как действует $h_{0} \in \mathscr{H}$ на $L_{-1}(\varepsilon)$. Напомним, что $L_{0}=M_{0} \oplus\left\langle h_{0}\right\rangle$, $\left\langle h_{0}\right\rangle$ - центр $L_{0}$. Если $v \in L_{-1}$, то $\left[v, h_{0}\right]=a v, a \in K^{*}$. Так как $L_{-1}(\varepsilon)=L_{-1}+\varepsilon L_{-1}-$ прямая сумма изоморфных неприводимых $M_{0}$-модулей, то $h_{0} \circ v=a v+\varepsilon b v=(a+\varepsilon b) v, a, b \in K, a, b$ не зависят от $v, a \neq 0$. Заменяя $h_{0}$ 
на $h_{0}-\varepsilon \frac{b}{a} h_{0}$, получим $h_{0} \circ v=a v$. Поэтому можно считать, что $\psi\left(h_{0}, L_{-1}\right)=0$. Так как $L_{-2}=\left[L_{-1}, L_{-1}\right]$ и $\psi\left(L_{-1}, L_{-1}\right)=0$, то из условия коцикла следует, что $\psi\left(h_{0}, L_{-2}\right)=0$.

Из тождества Якоби в $(L(\varepsilon)$, о $)$ следует, что для построенного элемента $h_{0}$ $\psi\left(h_{0}, L_{1}\right)=0$ и, следовательно, $\psi\left(h_{0}, L_{2}\right)=0$.

Итак, мы доказали, что коцикл $\psi$ когомологичен коциклу, удовлетворяюшему условиям $\psi\left(L_{0}, L_{i}\right)=0, i=0, \pm 1, \pm 2, \psi\left(L_{i}, L_{i}\right)=0, i=0, \pm 1$.

В дальнейшем нам понадобится понятие продолжений Картана. Пусть $L^{-}=$ $L_{-2}+L_{-1}+L_{0}, L_{-2}=\left[L_{-1}, L_{-1}\right]$ (в нашем случае $\operatorname{dim} L_{-2} \leqslant 1$ ) - транзитивная градуированная алгебра Ли, т.е. в $L_{0}$ выполняется условие: для $0 \neq$ $x \in L_{0},\left[x, L_{-1}\right] \neq 0$. Напомним, что множество $L^{(1)}$, состоящее из всех $\varphi \in$ $\operatorname{Hom}\left(L_{-1}, L_{0}\right) \oplus \operatorname{Hom}\left(L_{-2}, L_{-1}\right)$ таких, что $\left[\varphi\left(v_{1}\right), v_{2}\right]-\left[\varphi\left(v_{2}\right), v_{1}\right]=\varphi\left(\left[v_{1}, v_{2}\right]\right)$ для любых $v_{1}, v_{2} \in L_{-1}$, называется первым продолжсением Картана. $L^{(1)}$ имеет естественную структуру $L_{0}$-модуля: $\left(v_{0} \varphi\right)(v)=\left[v_{0}, \varphi(v)\right]-\varphi\left(\left[v_{0}, v\right]\right), v_{0} \in L_{0}$, $\varphi \in L^{(1)}, v \in L_{-i}, i=1,2$. Аналогично индукцией по $i$ определяются продолжения Картана $L^{(i)}, i>1$. На пространстве $L^{\infty}=L_{-2}+L_{-1}+L_{0}+L_{(1)}+L_{(2)}+\cdots$ имеется единственная структура алгебры Ли, относительно которой $L_{-2}+L_{-1}+L_{0}$ - подалгебра, изоморфная $L^{-}$, и $\left[\varphi, l_{j}\right]=\varphi\left(l_{j}\right)$ для $\varphi \in L^{(i)}, l_{j} \in L_{j}, j=-1,-2$.

Для классической алгебры Ли $L$ без центра, рассматриваемой вместе со стандартной градуировкой, можно считать, что $L_{1} \subset L^{(1)}$, отождествляя $w \in L_{1}$ с $\left.\operatorname{ad} w\right|_{L_{-1}} \in L^{(1)}$. Аналогично, $L_{2} \subset L^{(2)}$ и алгебра $L$ является однородной подалгеброй в $L^{\infty}$.

Лемма 4. Пусть $L$ - классическая алгебра одного из типов $\bar{A}_{l}, A_{l}^{z}, B_{l}$ $(l>2), C_{l} \quad(l>2), D_{l}, \bar{E}_{6}, E_{6}^{z}, E_{6} \quad(p>3), E_{7}, E_{8}, F_{4}$. B $L^{(1)}$ существует единственный $L_{0}$-подмодуль, изоморфный $L_{1}$.

ДокАЗАТЕЛьство. Пусть $\left\{\alpha_{1}, \ldots, \alpha_{l}\right\}$ - базис системы $R,\left\{\alpha_{2}, \ldots, \alpha_{l}\right\}$ - базис системы $R_{0}, \alpha_{1}$ связан в схеме Дынкина только с $\alpha_{2}$. Легко видеть, что младший вес $L_{0}$-модуля $L_{1}$ равен $\alpha_{1}$, а старший вес $L_{-1}$ равен $-\alpha_{1}$.

Допустим, что $\widehat{L}_{1}-L_{0}$-подмодуль в $L^{(1)}$, изоморфный $L_{1}, f$ - младший вектор подмодуля $\widehat{L}_{1}, f$ имеет вес $\alpha_{1}$. В алгебре Ли $L^{\infty}$ положим $\left[f, e_{-\alpha_{1}}\right]=h, h \in \mathscr{H}$. Если $h=0$, то $[f, v]=0$ для любого $v \in L_{-1}$, следовательно, $f=0$ и $\widehat{L}_{1}=0$. Если ранг $l$ больше 2 , то из тождества Якоби для $f, e_{-\alpha_{1}}, e_{\alpha_{i}}$ следует, что $\left[h, e_{\alpha_{i}}\right]=$ $\alpha_{i}(h) e_{\alpha_{i}}=0, i=3, \ldots, l$. Так как $L$ не имеет центра, то $\alpha_{3}, \ldots, \alpha_{l}$ линейно независимы как функции на $\mathscr{H}$. Следовательно, $h$ лежит в $\operatorname{ker} \alpha$ для $n-1$ линейно независимых функций $\alpha, \alpha=\alpha_{3}, \ldots, \alpha_{l}$. Пересечение ядер этих функций двумерно и натянуто на $h_{\alpha_{1}}, h_{0}$, где $h_{0}$ содержится в одномерном центре подалгебры $L_{0}$. Предположим, что $h$ не пропорционален $h_{0}$. Тогда, домножая при необходимости $f$ на константу, можем записать, что $\left[f, e_{\alpha_{-1}}\right]=h=h_{\alpha_{1}}+x h_{0}, x \in K$. Если ранг $l=2$, то полученное равенство сразу следует из того, что $\operatorname{dim} \mathscr{H} \leqslant 2$. Докажем, что $x=0$.

Так как $f$-младший вектор $\widehat{L}_{1}$, то $\left[e_{-\alpha_{2}}, f\right]=0$. Запишем тождество Якоби для $f, e_{-\alpha_{1}}, e_{-\alpha_{2}}$ :

$$
\begin{aligned}
{\left[\left[f, e_{-\alpha_{1}}\right], e_{-\alpha_{2}}\right]+\left[\left[e_{-\alpha_{1}}, e_{-\alpha_{2}}\right], f\right] } & =0 \\
{\left[h_{\alpha_{1}}+x h_{0}, e_{-\alpha_{2}}\right]+\left[\left[e_{-\alpha_{1}}, e_{-\alpha_{2}}\right], f\right] } & =0 .
\end{aligned}
$$


Так как $e_{-\alpha_{2}}$ принадлежит $L_{0}$, то $\left[h_{0}, e_{-\alpha_{2}}\right]=0$ и из последнего равенства получаем

$$
\left[h_{\alpha_{1}}, e_{-\alpha_{2}}\right]=\left[f,\left[e_{-\alpha_{1}}, e_{-\alpha_{2}}\right]\right]
$$

Из тождества Якоби для $e_{\alpha_{1}}, e_{-\alpha_{1}}, e_{-\alpha_{2}}$ следует, что

$$
\left[h_{\alpha_{1}}, e_{-\alpha_{2}}\right]=\left[e_{\alpha_{1}},\left[e_{-\alpha_{1}}, e_{-\alpha_{2}}\right]\right]
$$

Отсюда, так как $\left[e_{-\alpha_{1}}, e_{-\alpha_{2}}\right]=N e_{-\alpha_{1}-\alpha_{2}}, N \in K^{*}$,

$$
\left[f, e_{-\alpha_{1}-\alpha_{2}}\right]=\left[e_{\alpha_{1}}, e_{-\alpha_{1}-\alpha_{2}}\right]
$$

Запишем тождество Якоби для $f, e_{-\alpha_{1}}, e_{-\alpha_{1}-\alpha_{2}}$ :

$$
\left[\left[f, e_{-\alpha_{1}}\right], e_{-\alpha_{1}-\alpha_{2}}\right]-\left[\left[f, e_{-\alpha_{1}-\alpha_{2}}\right], e_{-\alpha_{1}}\right]=\left[f,\left[e_{-\alpha_{1}}, e_{-\alpha_{1}-\alpha_{2}}\right]\right]
$$

Отметим, что $\left[e_{-\alpha_{1}}, e_{-\alpha_{1}-\alpha_{2}}\right] \in L_{-2} ; L_{-2} \neq 0$ только для алгебр Ли типов $E_{7}$ и $E_{8}$ (см. табл. 1). В системах корней типа $E_{7}, E_{8}$ длина $\alpha$-серии корней, содержащей $\beta$, меньше 2 для любых $\alpha, \beta \in R$. Если $-\alpha_{1}+\left(-\alpha_{1}-\alpha_{2}\right)=-2 \alpha_{1}-\alpha_{2} \in R$, то длина $-\alpha_{1}$-серии, содержащей $-\alpha_{2}$, не менее 2 . Полученное противоречие показывает, что $\left[e_{-\alpha_{1}}, e_{-\alpha_{1}-\alpha_{2}}\right]=0$. Тогда из (8) получаем

$$
\left[\left[f, e_{-\alpha_{1}}\right], e_{-\alpha_{1}-\alpha_{2}}\right]-\left[\left[f, e_{-\alpha_{1}-\alpha_{2}}\right], e_{-\alpha_{1}}\right]=0
$$

Из (7) и того, что $\left[f, e_{-\alpha_{1}}\right]=h_{\alpha_{1}}+x h_{0}$, следует, что

$$
\left[h_{\alpha_{1}}+x h_{0}, e_{-\alpha_{1}-\alpha_{2}}\right]-\left[\left[e_{\alpha_{1}}, e_{-\alpha_{1}-\alpha_{2}}\right], e_{-\alpha_{1}}\right]=0
$$

Отсюда, используя тождество Якоби для $e_{\alpha_{1}}, e_{-\alpha_{1}}, e_{-\alpha_{1}-\alpha_{2}}$, получаем

$$
0=\left[h_{\alpha_{1}}, e_{-\alpha_{1}-\alpha_{2}}\right]+x\left[h_{0}, e_{-\alpha_{1}-\alpha_{2}}\right]-\left[\left[e_{\alpha_{1}}, e_{-\alpha_{1}}\right], e_{-\alpha_{1}-\alpha_{2}}\right]=x\left[h_{0}, e_{-\alpha_{1}-\alpha_{2}}\right]
$$

Так как $L$ не имеет центра, то $\left[h_{0}, e_{-\alpha_{1}-\alpha_{2}}\right] \neq 0$ и, следовательно, $x=0$.

Поэтому $\left[f, e_{-\alpha_{1}}\right]=h_{\alpha_{1}}=\left[e_{\alpha_{1}}, e_{-\alpha_{1}}\right]$. Так как $e_{\alpha_{-1}}-$ старший вектор $L_{-1}$, а $e_{\alpha_{1}}$ - младший вектор $L_{1}$, то из того, что $\left[f, e_{-\alpha_{1}}\right]=\left[e_{\alpha_{1}}, e_{-\alpha_{1}}\right]$, получаем, что $[f, v]=\left[e_{\alpha_{1}}, v\right]$ для любого $v$ из $L_{1}$. Следовательно, $f=e_{\alpha_{1}}, \widehat{L}_{1}=L_{1}$.

Осталось рассмотреть случай, когда $h$ пропорционален $h_{0}$. В этом случае можно считать, что $\left[f, e_{-\alpha_{1}}\right]=h_{0}$. Тогда из тождества Якоби для $f, e_{-\alpha_{1}}, e_{-\alpha_{2}}$ следует, что $\left[f, e_{-\alpha_{1}-\alpha_{2}}\right]=0$. Поэтому из $(9)$ получаем, что $0=\left[\left[f, e_{-\alpha_{1}-\alpha_{2}}\right], e_{-\alpha_{1}}\right]=$

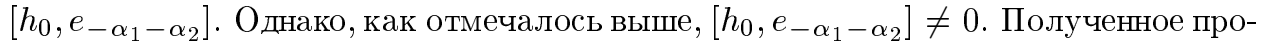
тиворечие показьвает, что $h$ не может быть пропорционален $h_{0}$.

ПРЕДЛОЖЕНИЕ 6 . Пусть $L-$ алгебра Ли одного из типов $\bar{A}_{l}, A_{l}^{z}, B_{l}(l>2)$, $C_{l}(l>2), D_{l}, \bar{E}_{6}, E_{6}^{z}, E_{6} \quad(p>3), E_{7}, E_{8}, F_{4}$. Eсли $\psi \in Z_{0}^{2}(L, L)$ такой, что $\psi\left(L_{0}, L_{i}\right)=0, i=0, \pm 1, \pm 2, \psi\left(L_{i}, L_{i}\right)=0, i=0, \pm 1$, то $\psi$ когомологичен нулю. 
ДокАЗАТЕльство. Для доказательства предложения достаточно установить, что помимо условий, накладываемых на коцикл $\psi$, выполняются также условия: $\psi\left(L_{-i}, L_{i}\right)=0, i=1,2$ и и $\psi\left(L_{-1}, L_{2}\right)=\psi\left(L_{1}, L_{-2}\right)=0$.

Для $v_{1}, v_{2} \in L_{-1}, w \in L_{1} w \circ v_{i} \in L_{0}(\varepsilon), i=1,2$. Так как $\psi\left(v, v_{0}\right)=0$ для $v_{0} \in L_{0}, v \in L_{-1}$, то в $L(\varepsilon)$

$$
\left(v_{1} \circ v_{2}\right) \circ w=\left[v_{1},\left[v_{2}, w\right]\right]+\varepsilon\left[v_{1}, \psi\left(v_{2}, w\right)\right]-\left[v_{2},\left[v_{1}, w\right]\right]-\varepsilon\left[v_{2}, \psi\left(v_{1}, w\right)\right] .
$$

Если $L_{-2}=0$, то

$$
\left[v_{1}, \psi\left(v_{2}, w\right)\right]-\left[v_{2}, \psi\left(v_{1}, w\right)\right]=0
$$

Если $L_{-2} \neq 0$, то из условий на коцикл $\psi$ получаем $v_{1} \circ v_{2}=\left[v_{1}, v_{2}\right]=\left\langle v_{1}, v_{2}\right\rangle e_{\gamma}$, где $\left\langle v_{1}, v_{2}\right\rangle \in K,\left\langle e_{\gamma}\right\rangle=L_{-2}$. Из (10) следует

$$
\left[v_{1}, \psi\left(v_{2}, w\right)\right]-\left[v_{2}, \psi\left(v_{1}, w\right)\right]=\left\langle v_{1}, v_{2}\right\rangle \psi\left(e_{\gamma}, w\right)
$$

Тождества (11), (12) означают, что $\psi(w,-)$ принадлежит продолжению Картана $L^{(1)}$.

Обозначим через $\widehat{L}_{1}$ подпространство в $L^{(1)}$, состоящее из отображений $\psi(w,-)$, $w \in L_{1}$. Из соотношения $d \psi\left(l_{0}, v, w\right)=0, l_{0} \in L_{0}, v \in L_{-1}, w \in L_{1}$, и условий на коцикл $\psi$ следует, что

$$
l_{0} \psi(w,-)=\psi\left(\left[l_{0}, w\right],-\right) .
$$

Таким образом, $\widehat{L}_{1}-L_{0}$-подмодуль в $L^{(1)}$ и соответствие $w \mapsto \psi(w,-)$ является морфизмом $L_{0}$-подмодулей $L_{1}$ и $\widehat{L}_{1}$. Допустим, что $\widehat{L}_{1} \neq 0$. Тогда $\widehat{L}_{1}-$ неприводимьй $L_{0}$-модуль и $L_{0}$-модули $L_{1}$ и $\widehat{L}_{1}$ изоморфны. Из леммы 4 получаем, что $L_{1}=\widehat{L}_{1}$. Более того, по лемме Шура сушествует $c \in K, c \neq 0$, такое, что $c w=\psi(w,-)$ для всех $w \in L_{1}$. Следовательно, для $v \in L_{-1}, w \in L_{1}$ в $L(\varepsilon)$ имеем $v \circ w=[v, w]+\varepsilon c[v, w]$. Заменяя $\psi$ на $\psi+\delta \varphi$, где $\varphi(v)=c v$, если $v \in L_{-1}$, $\varphi(v)=2 c v$, если $v \in L_{-2}$, и $\left.\varphi\right|_{L_{0} \oplus L_{1} \oplus L_{2}}=0$, получаем, что $\psi\left(L_{-1}, L_{1}\right)=0$.

Предположим, что $L_{ \pm 2} \neq 0, v_{1}, v_{2} \in L_{-1}, v \in L_{1}$. Из условий на коцикл $\psi$ и полученного условия $\psi\left(L_{-1}, L_{1}\right)=0$ следует, что

$$
0=d \psi\left(v_{1}, v_{2}, v\right)=-\psi\left(\left[v_{1}, v_{2}\right], v\right)
$$

Так как $\left[L_{-1}, L_{-1}\right]=L_{-2}$, то $\psi\left(L_{-2}, L_{1}\right)=0$. Аналогично, $\psi\left(L_{2}, L_{-1}\right)=0$. Если $w \in L_{2}$, то из условий на коцикл $\psi$ следует

$$
0=d \psi\left(v_{1}, v_{2}, w\right)=-\psi\left(\left[v_{1}, v_{2}\right], w\right)
$$

Поэтому $\psi\left(L_{-2}, L_{2}\right)=0$.

Если $L$ имеет центр, то $L=A_{l}, l+1 \equiv 0(p)$, или при $p=3 L=E_{6}$. В этих алгебрах $L_{ \pm 2}=0$.

Согласно предложению 1 мы можем не рассматривать алгебры Ли типа $A_{l}$, когда $l+1 \not \equiv 0(p)$, так как в этом случае форма Киллинга невырождена (см. табл. 1). 
ПРЕДЛОЖЕНИЕ 7. Пусть $L$ - алгебра Ли одного из типов $A_{l}, l+1 \equiv 0(p)$, или $E_{6}(p=3), \psi \in Z_{0}^{2}(L, L)$. Если $\psi\left(M_{0}, L_{ \pm 1}\right)=0, \psi\left(L_{i}, L_{i}\right)=0, i=0, \pm 1$, то $\psi$ когомологичен нулю.

Пусть $\left\{\alpha_{1}, \ldots, \alpha_{l}\right\}$ - базис системы $R$, a $\left\{\alpha_{2}, \ldots, \alpha_{l}\right\}$ - базис системы $R_{0}$.

Лемма 5. (а) $W\left(R_{0}\right) \alpha_{1}=R_{1}$;

(b) ecлu $\alpha \in R_{1}, \beta \in R_{0}, \alpha+\beta \in R$, mo $h_{\alpha+\beta}=h_{\alpha}+h_{\beta}$.

ДокАЗАтЕльство. Пусть $\alpha, \beta \in R, \beta-q \alpha, \ldots, \beta+r \alpha, q, r \in \mathbb{Z}^{+},-\alpha$-серия корней, содержащая $\beta, p+q$-ее длина. Так как все корни в системах типа $A_{l}, E_{6}$ имеют одинаковую длину, то из [11; гл. VI, $\S 1$ п. 3 , замечание к предложению 9] следует, что $r+q \leqslant 1$. Поэтому если $\alpha+\beta \in R$, то $q=0, r=1$ и $\langle\alpha, \beta\rangle=\frac{2(\alpha, \beta)}{(\beta, \beta)}=$ $q-r=-1$. Следовательно, $\alpha+\beta=\alpha-\langle\alpha, \beta\rangle \beta=w_{\beta} \alpha$.

(a) Любой корень $\beta \neq \alpha_{1}$ из $R_{1}$ мы можем получить последовательным прибавлением к $\alpha_{1}$ корней из базиса $R_{0}$, при этом на каждом шаге получаем корни из $R_{1}$. Из приведенного вьше рассуждения следует, что $\beta=w_{0} \alpha_{1}, w_{0} \in W\left(R_{0}\right)$.

(b) Напомним, как группа $W$ действует на $\mathscr{H}: w_{\alpha_{i}} h_{\alpha_{j}}=h_{\alpha_{j}}-\left\langle\alpha_{i}, \alpha_{j}\right\rangle h_{\alpha_{i}}$ (см., например, [14]). Так как все корни системы $R$ имеют одинаковую длину, то $\left\langle\alpha_{i}, \alpha_{j}\right\rangle=\left\langle\alpha_{j}, \alpha_{i}\right\rangle$ и $w_{\alpha_{i}} h_{\alpha_{j}}=h_{\alpha_{j}}-\left\langle\alpha_{j}, \alpha_{i}\right\rangle h_{\alpha_{i}}$. Мы видим, что если $w_{\alpha_{i}} \alpha_{j}=\alpha_{j}-$ $\left\langle\alpha_{j}, \alpha_{i}\right\rangle \alpha_{i}=\alpha_{j}+\alpha_{i}$, то $w_{\alpha_{i}} h_{\alpha_{j}}=h_{\alpha_{j}}+h_{\alpha_{i}}$. Согласно (а) для любого $\alpha \in R_{1}$ сушествует $w \in W\left(R_{0}\right)$ такой, что $w \alpha_{1}=\alpha$. Так как $W(R)$ порождается $w_{\alpha_{i}}$, $i=1, \ldots, l$, то $w \alpha_{1}=x_{1} \alpha_{1}+\cdots+x_{l} \alpha_{l}-$ разложение $\alpha$ по базису $R$. Действие $w_{\alpha_{i}}$ на $\alpha_{j}$ полностью аналогично действию $w_{\alpha_{i}}$ на $h_{\alpha_{j}}$. Поэтому если $\alpha=x_{1} \alpha_{1}+\cdots+$ $x_{l} \alpha_{l}$ - разложение $\alpha$ по базису $R$, то $x_{1} h_{\alpha_{1}}+\cdots+x_{l} h_{\alpha_{l}}$ - разложение $h_{\alpha}$ по $h_{\alpha_{i}}$. Отсюда если $\alpha+\beta \in R$, то $h_{\alpha+\beta}=h_{\alpha}+h_{\beta}$.

Из леммы 5 следует, что для $\alpha \in R_{1}$ сушествует $w_{0} \in W\left(R_{0}\right)$ такой, что $\alpha=$ $w_{0} \alpha_{1}$. Тогда $h_{\alpha}=h_{w_{0} \alpha_{1}}=w_{0} h_{\alpha_{1}}=h_{\alpha_{1}}+h$, где $h \in \mathscr{H}_{0}$. Так как $\mathscr{H}=\mathscr{H}_{0} \oplus I$, где $I$ - одномерный центр $L$, то $h_{\alpha_{1}}=h\left(\alpha_{1}\right)-h_{0}, h\left(\alpha_{1}\right) \in \mathscr{H}_{0}, h_{0} \in I$. Следовательно, для любого $\alpha \in R_{1} h_{\alpha}$ представляется в виде: $h_{\alpha}=h(\alpha)-h_{0}$, где $h(\alpha) \in \mathscr{H}_{0}$, т.е. компонента $h_{\alpha}$ в $I$ не зависит от $\alpha$.

Любой корень $\gamma$ из $\left\{\alpha_{3}, \ldots, \alpha_{l}\right\} \subset R_{0}$ ортогонален к $\alpha_{1}$ и $\gamma+\alpha_{1}, \gamma-\alpha_{1}$ не являются корнями. Теперь из п. (а) леммы 5 следует, что для любого $\alpha \in R_{1}$ сушествует ровно $l-2$ линейно независимых корня $\gamma \in R_{0}$ таких, что $\gamma+\alpha, \gamma-\alpha$ не являются корнями. Если $\gamma+\alpha \notin R, \gamma-\alpha \notin R$, то $e_{\alpha} \circ e_{\gamma}=e_{-\alpha} \circ e_{\gamma}=0$. Для $\alpha \in R_{1}$

$$
e_{\alpha} \circ e_{-\alpha}=h_{\alpha}+\varepsilon H_{\alpha}
$$

где $H_{\alpha}$ - некоторый элемент из $\mathscr{H}$. Так как $e_{\gamma} \in L_{0}$ и $\psi\left(L_{0}, L_{0}\right)=0$, то из тождества Якоби для $e_{\alpha}, e_{-\alpha}, e_{\gamma}$ следует, что $\left[H_{\alpha}, e_{\gamma}\right]=\gamma\left(H_{\alpha}\right) e_{\gamma}=0$. Таким образом, $H_{\alpha}$ содержится в пересечении ядер $l-2$ линейно независимых функций $\gamma$. Так как $h(\alpha)$ и $h_{0}$ также содержатся в этом пересечении, то $H_{\alpha}=x_{\alpha} h(\alpha)+y_{\alpha} h_{0}$, где $x_{\alpha}, y_{\alpha} \in K$. Таким образом,

$$
e_{\alpha} \circ e_{-\alpha}=h(\alpha)-h_{0}+\varepsilon\left(x_{\alpha} h(\alpha)+y_{\alpha} h_{0}\right)
$$


Лемма 6. (а) Пусть $R$ - система корней типа $A_{l}, l>2, l+1 \equiv 0(p)$, или $E_{6}(p=3), \alpha, \beta \in R_{1}$. Eсли существует простой корень $\gamma \in R_{0}$ такой, что $\alpha=\beta+\gamma$, mo $x_{\alpha}=x_{\beta}, y_{\alpha}=y_{\beta}$.

(b) Eсли $R$ - система типа $A_{2}$, mо $x_{\alpha}=x_{\beta}, y_{\alpha}=y_{\beta}$ для любъх $\alpha, \beta \in R_{1}$.

ДокаЗАтЕЛьство. (а) Из п. (b) леммы 5 следует, что $h_{\alpha}=h_{\beta}+h_{\gamma}$. Поэтому $h(\alpha)=h(\beta)+h_{\gamma}$. Пусть $\left[e_{\gamma}, e_{-\alpha}\right]=B e_{-\beta},\left[e_{\gamma}, e_{\beta}\right]=A e_{\alpha}, e_{\beta} \circ e_{-\alpha}=C e_{-\gamma}$, где $A, B \in K^{*}, C$ - обратимьй элемент из $K[\varepsilon]$. Так как $e_{\gamma} \in L_{0}$, то из тождества Якоби

$$
e_{\gamma} \circ\left(e_{\beta} \circ e_{-\alpha}\right)=\left(e_{\gamma} \circ e_{\beta}\right) \circ e_{-\alpha}+e_{\beta} \circ\left(e_{\gamma} \circ e_{-\alpha}\right)
$$

и условий на коцикл $\psi$ следует, что

$$
C h_{\gamma}=A e_{\alpha} \circ e_{-\alpha}+B e_{\beta} \circ e_{-\beta}
$$

Из (13) получаем, что

$$
C h_{\gamma}=A\left(h(\alpha)-h_{0}+\varepsilon\left(x_{\alpha} h(\alpha)+y_{\alpha} h_{0}\right)\right)+B\left(h(\beta)-h_{0}+\varepsilon\left(x_{\beta} h(\beta)+y_{\beta} h_{0}\right)\right) .
$$

Так как $h(\alpha)=h(\beta)+h_{\gamma}$, то

$$
\begin{aligned}
C h_{\gamma}= & (B+A) h(\beta)-(B+A) h_{0}+A h_{\gamma}+\varepsilon\left(B x_{\beta}+A x_{\alpha}\right) h(\beta) \\
& +\varepsilon\left(B y_{\beta}+A y_{\alpha}\right) h_{0}+\varepsilon A x_{\alpha} h_{\gamma} .
\end{aligned}
$$

Докажем, что в разложение $h(\beta)$ по $h_{\alpha_{i}}, \alpha_{i}$ - простые корни системы $R_{0}, \mathrm{c}$ ненулевым коэффициентом входит хотя бы один $h_{\alpha_{i}}, \alpha_{i} \neq \gamma$, и, следовательно,

$$
\left\{\begin{array}{l}
A+B=0 \\
B x_{\beta}+A x_{\alpha}=0 \\
B y_{\beta}+A y_{\alpha}=0
\end{array}\right.
$$

Отсюда $x_{\alpha}=x_{\beta}, y_{\alpha}=y_{\beta}$.

Предположим, что $h(\beta)=x h_{\gamma}$, где $x \in K^{*}, \gamma$ - простой корень системы $R_{0}$. Тогда $h_{0}=h(\beta)-h_{\beta}=x h_{\gamma}-h_{\beta}$. Следовательно, $(x \gamma-\beta)\left(h_{\alpha}\right)=0$ для любого $\alpha \in R$.

Рассмотрим векторное пространство над $K$

$$
V_{K}=Q(R) \otimes_{\mathbb{Z}} K
$$

с базисом $\left\{\alpha_{1}, \ldots, \alpha_{l}\right\}$. Предположим, что $x \gamma=\beta$. Так как $\beta \in R_{-1}, \gamma \in R_{0}$, то $\beta=\alpha_{1}-\alpha_{0}, \alpha_{0}, \gamma \in\left\langle\alpha_{2}, \ldots, \alpha_{l}\right\rangle_{K}$. Тогда $\alpha_{1}=x \gamma+\alpha_{0} \in\left\langle\alpha_{2}, \ldots, \alpha_{l}\right\rangle_{K}$. Получили противоречие, следовательно, $x \gamma-\beta \neq 0$.

Обозначим подпространство в $V_{\mathbb{R}}$, натянутое на $\gamma, \beta$, через $U$. Так как корни $\gamma$ и $\beta$ линейно независимы над $\mathbb{R}$, то $R_{U}=R \cap U$ является системой корней ранга 2. Согласно [11; гл. VI, $\S 1$, п. 7, предложение 24$]$ существует базис $\left\{\delta_{1}, \ldots, \delta_{l}\right\}$ системы $R$, содержащий базис системы $R_{U}$. Будем считать, что $R_{U}$ имеет базис $\left\{\delta_{1}, \delta_{2}\right\}$. По-прежнему $\left\langle\delta_{1}, \ldots, \delta_{l}\right\rangle_{K}=V_{K}$. Определим спаривание $\langle\cdot, \cdot\rangle$ пространств $V_{K}$ и $\mathscr{H}$, полагая $\left\langle\delta_{i}, h_{\delta_{j}}\right\rangle=\left\langle\delta_{i}, \delta_{j}\right\rangle=a_{i j}, a_{i j}$ - числа Картана. Обозначим через $V_{1}$ 
ядро спаривания. Поскольку $(x \gamma-\beta)\left(h_{\alpha}\right)=0$ для всех $\alpha$ из $R$, то $x \gamma-\beta \in V_{1}$. Векторы $\gamma, \beta$ раскладываются по $\delta_{1}, \delta_{2}$ и $\mu=x \gamma-\beta=x_{1} \delta_{1}+x_{2} \delta_{2}$. Если $x_{2}=0$, то $0=\left\langle\mu, h_{\delta_{1}}\right\rangle=x_{1}\left\langle\delta_{1}, \delta_{1}\right\rangle$. Следовательно, $x_{1}=0$. Аналогично, если $x_{1}=0$, то $x_{2}=0$. Однако $x \gamma-\beta \neq 0$, поэтому $x_{1}, x_{2} \in K^{*}$. Если $\delta_{1}$ не связан в схеме Дынкина с $\delta_{2}$, то $0=\left\langle\mu, h_{\delta_{1}}\right\rangle=x_{1}\left\langle\delta_{1}, \delta_{1}\right\rangle$, следовательно, $x_{1}=0$. Получили противоречие, значит, $\delta_{1}$ связан с $\delta_{2}$ в схеме Дынкина и $R_{U}$ - система типа $A_{2}$. Так как ранг $l>2$, то сушествует $i$ такой, что $\delta_{i}$ связан в схеме Дынкина только с одним из $\delta_{1}, \delta_{2}$ (можно считать, что с $\left.\delta_{1}\right)$. Тогда $0=\left\langle\mu, h_{\delta_{i}}\right\rangle=x_{1}\left\langle\delta_{1}, \delta_{i}\right\rangle$. Получили противоречие.

(b) Рассмотрим случай $A_{2}, p=3$.

Пусть система корней $R$ типа $A_{2}$ имеет базис $\left\{\alpha_{1}, \alpha_{2}\right\}, R_{0}$ имеет базис $\left\{\alpha_{1}\right\}$. Тогда $h_{0}=h_{\alpha_{1}}-h_{\alpha_{2}}, h_{\alpha_{1}+\alpha_{2}}=h_{\alpha_{1}}+h_{\alpha_{2}}=-h_{\alpha_{1}}-h_{0}$,

$$
\begin{aligned}
e_{\alpha_{2}} \circ e_{-\alpha_{2}} & =\left(1+\varepsilon x_{\alpha_{2}}\right) h_{\alpha_{1}}-\left(1-\varepsilon y_{\alpha_{2}}\right) h_{0}, \\
e_{\alpha_{1}+\alpha_{2}} \circ e_{-\alpha_{1}-\alpha_{2}} & =-\left(1+\varepsilon x_{\alpha_{1}+\alpha_{2}}\right) h_{\alpha_{1}}-\left(1-\varepsilon y_{\alpha_{1}+\alpha_{2}}\right) h_{0} .
\end{aligned}
$$

Из тождества Якоби для $e_{\alpha_{1}}, e_{\alpha_{2}}, e_{-\alpha_{1}-\alpha_{2}}$ и тождества Якоби для $e_{-\alpha_{1}-\alpha_{2}}$, $e_{\alpha_{2}}, e_{-\alpha_{2}}$ легко получить, что $x_{\alpha_{1}+\alpha_{2}}=x_{\alpha_{2}}$ и $y_{\alpha_{1}+\alpha_{2}}=y_{\alpha_{2}}$.

Теперь, так как любой корень из $R_{1}$ может быть включен в цепочку корней, получающуюся последовательным прибавлением к $\alpha_{1}$ простых корней из $R_{0}$, то из леммы 6 следует, что $x_{\alpha}=x_{\beta}=x$ и $y_{\alpha}=y_{\beta}=y$ для любых $\alpha, \beta \in R_{1}$.

Преобразуем (13) следуюшим образом:

$$
e_{\alpha} \circ e_{-\alpha}=(1+\varepsilon x)\left(h(\alpha)-\frac{1-\varepsilon y}{1+\varepsilon x} h_{0}\right) .
$$

Заменяя базис $\left\{e_{\alpha}, \alpha \in R_{1}\right\}$ в $L_{1}$ на $\left\{(1-\varepsilon x) e_{\alpha}\right\}$ и заменяя $h_{0}$ на $\frac{1-\varepsilon y}{1+\varepsilon x} h_{0}$, получим из (14):

$$
e_{\alpha} \circ e_{-\alpha}=h(\alpha)-h_{0}=h_{\alpha}
$$

для любого $\alpha \in R_{1}$. Поэтому мы можем считать, что $\psi\left(e_{\alpha}, e_{-\alpha}\right)=0$.

Если $\alpha-\beta \notin R$ для $\alpha \in R_{1},-\beta \in R_{-1}$, то $\psi\left(e_{\alpha}, e_{-\beta}\right)=0$. Если $\alpha_{0}=\alpha-\beta \in R_{0}$, то $\alpha=\beta+\alpha_{0}$. Так как длина $\beta$-последовательности, содержащей $\alpha_{0}$, не больше 1 , то $\alpha_{0}-\beta \notin R$. Тогда

$$
0=d \psi\left(e_{\beta}, e_{-\beta}, e_{\alpha_{0}}\right)=\psi\left(\left[e_{\beta}, e_{\alpha_{0}}\right], e_{-\beta}\right)=c \psi\left(e_{\alpha}, e_{-\beta}\right),
$$

где $\left[e_{\beta}, e_{\alpha_{0}}\right]=c e_{\alpha}, c \in K^{*}$. Следовательно, $\psi\left(e_{\alpha}, e_{-\beta}\right)=0$ для любых $\alpha, \beta \in K$ и $\psi\left(L_{-1}, L_{1}\right)=0$.

Пусть $\alpha, \beta \in R_{1}$. Из условий на коцикл $\psi$ следует, что

$$
0=d \psi\left(e_{\alpha}, e_{-\alpha}, e_{\beta}\right)=-\psi\left(\left[e_{\alpha}, e_{-\alpha}\right], e_{\beta}\right)=-\psi\left(h_{\alpha}, e_{\beta}\right) .
$$

Следовательно, $\psi\left(\mathscr{H}, L_{1}\right)=0$. Аналогично, $\psi\left(\mathscr{H}, L_{-1}\right)=0$. Объединяя все условия, полученные на коцикл $\psi$, имеем $\psi(L, L)=0$.

Итак, мы установили, что любой коцикл $\psi$ нулевого веса когомологичен нулю и, следовательно, $H_{0}^{2}(L, L)=0$. Объединяя это с результатами $\S 3$, получаем, что все классические алгебры Ли над полем характеристики $p>2$ являются жесткими, кроме алгебры типа $C_{2}(p=3)$. 


\section{Список литературы}

1. Рудаков А. Н. Деформации простых алгебр Ли // Изв. АН СССР. Сер. матем. 1971. T. 35. C. 1113-1119.

2. Brown G. Lie algebras of characteristic three with non-degenerate Killing form // Trans. Amer. Math. Soc. 1969. V. 137. P. 259-268.

3. Кострикин А. И. Параметрическое семейство простых алгебр Ли // Изв. АН СССР. Сер. матем. 1970. Т. 34. С. 744-756.

4. Джумадильдаев А. С. К деформациям классических простых алгебр Ли // УМН. 1976. T. 31. № 3. C. 211-212.

5. Кострикин А.И., Кузнецов М.И. О деформациях классических алгебр Ли характеристики три // Докл. РАН. 1995. Т. 343. № 3. С. 299-301.

6. Кириллов С. А., Кузнецов М.И., Чебочко Н. Г. О деформациях алгебры Ли типа $G_{2}$ характеристики три // Изв. вузов. Матем. В печати.

7. Gerstenhaber M. On the deforormation of rings and algebras // Ann. of Math. (2). 1964. V. 79. №1. P. 59-103.

8. Frohardt D.E., Griess R.L.(Jr.). Automorphisms of modular Lie algebras // Nova J. Algebra Geom. 1992. V. 1. P. 339-345.

9. Спрингер T. А., Штейнберг Р. Классы сопряженных элементов // Сб. трудов семинара по алгебраическим группам. М.: Мир, 1973. С. 162-262.

10. Seligman G. B. Modular Lie algebras. New York: Springer-Verlag, 1967.

11. Бурбаки Н. Группы и алгебры Ли. Гл. IV-VI. М.: Мир, 1972.

12. Бурбаки Н. Группы и алгебры Ли. Гл. VII-VIII. М.: Мир, 1978.

13. Кострикин А.И., Острик В.В.К теореме распознавания для алгебр Ли характеристики 3 // Матем. сб. 1995. Т. 186. № 10. С. 73-88.

14. Стейнберг Р. Лекции о группах Шевалле. М.: Мир, 1975.

Нижегородский государственный

Поступила в редакцию

университет им. Н.И. Лобачевского

21.10 .1999 